\title{
ON THE STABILITY OF AXIALLY SYMMETRIC EQUILIBRIUM FIGURES OF A ROTATING VISCOUS INCOMPRESSIBLE FLUID
}

\author{
V. A. SOLONNIKOV
}

\begin{abstract}
It is proved that if the second variation of the energy functional $R$ (see $(2.9))$ is positive, then the axially symmetric equilibrium figure of a viscous incompressible capillary fluid is stable. The proof is based on the study of a nonstationary free boundary problem for the Navier-Stokes system with initial data close to the rotation regime of the fluid as a rigid body.
\end{abstract}

\section{$\S 1$. INTRODUCTION}

Unsteady motion of an isolated liquid mass under the surface tension force on the boundary and the self-gravity forces is governed by the following problem: to find a bounded domain $\Omega_{t} \subset \mathbb{R}^{3}$, a vector field $\vec{v}(x, t)=\left(v_{1}, v_{2}, v_{3}\right)$, and a function $p(x, t)$, $x \in \Omega_{t}$, satisfying the relations

$$
\begin{gathered}
\vec{v}_{t}+(\vec{v} \cdot \nabla) \vec{v}-\nu \nabla^{2} \vec{v}+\nabla p=0, \quad \nabla \cdot \vec{v}=0, \quad x \in \Omega_{t}, \quad t>0, \\
\left.\vec{v}\right|_{t=0}=\vec{v}_{0}(x), \quad x \in \Omega_{0}, \\
T(\vec{v}, p) \vec{n}=(\sigma H+\varkappa U(x, t)) \vec{n}, \quad V_{n}=\vec{v} \cdot \vec{n}, \quad x \in \Gamma_{t} \equiv \partial \Omega_{t}, \quad t>0,
\end{gathered}
$$

where $\Omega_{0}$ is a given domain, $\vec{n}$ is the outward unit normal to the boundary $\Gamma_{t}$ of the domain $\Omega_{t}, H$ is twice the mean curvature of $\Gamma_{t}, U(x, t)=\int_{\Omega_{t}} \frac{d y}{|x-y|}$ is the Newtonian potential, and $T(v, p)=-p I+\nu S(\vec{v})$ and $S(v)=\left(\frac{\partial v_{i}}{\partial x_{j}}+\frac{\partial v_{j}}{\partial x_{i}}\right)_{i, j=1,2,3}$ are the stress tensor and twice the rate-of-strain tensor, respectively; $I$ is the identity tensor. The coefficients $\nu, \sigma, \varkappa$ are given positive constants, but the constant $\varkappa$ can also be set equal to zero, which corresponds to the absence of self-gravitation. By $V_{n}$ we denote the velocity of the motion of $\Gamma_{t}$ in the direction of $\vec{n}$.

Suppose a drop of a liquid rotates as a rigid body about the axis $x_{3}$ with constant angular speed $\omega_{0}$. The velocity and pressure are determined by the formulas

$$
\begin{aligned}
& \overrightarrow{\mathcal{V}}(x)=\omega_{0}\left(-x_{2}, x_{1}, 0\right)=\omega_{0} \vec{e}_{3} \times \vec{x}, \\
& \mathcal{P}(x)=\frac{1}{2}|\overrightarrow{\mathcal{V}}(x)|^{2}+p_{0}=\frac{\omega_{0}^{2}}{2}\left(x_{1}^{2}+x_{2}^{2}\right)+p_{0},
\end{aligned}
$$

where $\vec{e}_{3}=(0,0,1)$ and $p_{0}=$ const, while the boundary conditions reduce to a single equation

$$
\sigma \mathcal{H}+\mathcal{P}+\varkappa \mathcal{U}=0
$$

on the surface of the rotating liquid. The domain filled by the rotating liquid is called an equilibrium figure and is denoted by $\mathcal{F}$; its boundary is denoted by $\mathcal{G}$. In (1.2), by $\mathcal{H}$

2000 Mathematics Subject Classification. Primary 35Q30.

Key words and phrases. Equilibrium figures, free boundary problems, stability.

Supported by RFBR (grant no. 03-01-00638). 
we mean twice the mean curvature of $\mathcal{G}$, and

$$
\mathcal{U}(x)=\int_{\mathcal{F}} \frac{d y}{|x-y|}
$$

Equation (1.2) serves for determining $\mathcal{G}$. If $\mathcal{F}$ is axially symmetric with respect to the axis $x_{3}$, then $(\mathcal{F}, \overrightarrow{\mathcal{V}}(x), \mathcal{P}(x))$ is a stationary solution of problem (1.1). Clearly, the full angular momentum

$$
\vec{\beta}=\int_{\mathcal{F}}(\vec{x} \times \overrightarrow{\mathcal{V}}(x)) d x
$$

is parallel to the axis $x_{3}, \vec{\beta}=\beta \vec{e}_{3} ; \beta$ and $\omega_{0}$ are related as follows:

$$
\beta=\omega_{0} \mathcal{I}, \quad \mathcal{I}=\int_{\mathcal{F}}\left(x_{1}^{2}+x_{2}^{2}\right) d x .
$$

In addition, we assume that the barycenter of $\mathcal{F}$ coincides with the origin, i.e.,

$$
\int_{\mathcal{F}} x_{i} d x=0, \quad i=1,2,3 .
$$

Equation (1.2) is the Euler equation for the functionals

$$
G=\sigma|\Gamma|-\frac{\omega_{0}^{2}}{2} \int_{\Omega}\left(x_{1}^{2}+x_{2}^{2}\right) d x-\frac{\varkappa}{2} \int_{\Omega} U(x) d x-p_{0}|\Omega|,
$$

where $U(x)=\int_{\Omega}|x-y|^{-1} d y,|\Gamma|=$ meas $\Gamma,|\Omega|=$ meas $\Omega$, and

$$
R=\sigma|\Gamma|+\frac{\beta^{2}}{2 \int_{\Omega}\left(x_{1}^{2}+x_{2}^{2}\right) d x}-\frac{\varkappa}{2} \int_{\Omega} U(x) d x-p_{0}|\Omega| ;
$$

these functionals are defined on a set of domains $\Omega$ that are close to $\mathcal{F}$ and have the same volume and barycenter as $\mathcal{F} ; \Gamma=\partial \Omega$. An arbitrary surface close to $\mathcal{G}$ can be given by an equation of the form

$$
x=y+N(y) \rho(y), \quad y \in \mathcal{G},
$$

where $N(y)$ is the outward normal to $\mathcal{G}$ and $\rho(y)$ is some small function,

$$
|\rho|_{C^{1}(\mathcal{G})} \leq \delta \ll 1,
$$

so that $G$ and $R$ can be viewed as $\rho$-dependent functionals. It is easy to verify that

$$
\delta_{0} G[r]=\delta_{0} R[r]=-\int_{\mathcal{G}}(\sigma \mathcal{H}+\mathcal{P}+\varkappa \mathcal{U}(x)) r(x) d S=0,
$$

where

$$
\delta_{0} G[r]=\left.\frac{\partial}{\partial \lambda} G[\rho+\lambda r]\right|_{\rho=0, \lambda=0} .
$$

As to the stability of $\mathcal{F}$, the universally recognized stability condition is that the second variations of these functionals should be positive; if we deal with free motion of the fluid with preservation of the angular momentum, then the condition

$$
\delta_{0}^{2} R[r]=\left.\frac{\partial^{2}}{\partial \lambda^{2}} R[\rho+\lambda r]\right|_{\lambda=0, \rho=0}>0
$$

is more natural (see [1]-[3]).

Comparison of (1.4) and (1.5) shows that

$$
\delta_{0}^{2} R-\delta_{0}^{2} G=\frac{\beta^{2}}{2} \delta_{0}^{2} I^{-1}+\frac{\beta^{2}}{2 \mathcal{I}^{2}} \delta_{0}^{2} I=\frac{\beta^{2}}{\mathcal{I}^{3}}\left(\delta_{0} I\right)^{2}>0,
$$


where $I=\int_{\Omega}\left(x_{1}^{2}+x_{2}^{2}\right) d x$. Therefore, if $\delta_{0}^{2} G$ is positive, then so is $\delta_{0}^{2} R$. We assume that an equilibrium figure is stable if, under initial data $\left(\Omega_{0}, \vec{v}_{0}\right)$ close to $(\mathcal{F}, \overrightarrow{\mathcal{V}}(x))$ and such that

$$
\begin{gathered}
\int_{\Omega_{0}} \vec{v} d x=0, \quad \int_{\Omega_{0}}\left(\vec{x} \times \vec{v}_{0}(x)\right) d x=\beta \vec{e}_{3}, \\
\left|\Omega_{0}\right|=|\mathcal{F}|, \quad \int_{\Omega_{0}} x_{i} d x=0, \quad i=1,2,3,
\end{gathered}
$$

problem (1.1) has a solution defined for all $t>0$ and satisfying $\vec{v}(x, t)-\overrightarrow{\mathcal{V}}(x) \rightarrow 0$, $p(x, t)-\mathcal{P}(x) \rightarrow 0$, and $\Gamma_{t} \rightarrow \mathcal{G}$ as $t \rightarrow \infty$.

In [4]-6], the stability of the axially symmetric equilibrium figures was proved under the condition $\delta_{0}^{2} G>0$ (for $\varkappa=0$ in [4, 5] and for $\varkappa>0$ in [6]). Here we do the same under the more natural condition (1.8), which has required several modifications in the proofs. Moreover, we remedy some inaccuracies occurred in [5, 6]. The main result is formulated in Theorem 2.1.

\section{§2. Auxiliary Relations and statement of the main Result}

Assume that equation (1.2), which can also be written in the form

$$
\sigma \mathcal{H}(y)+\frac{\beta^{2}}{2 \mathcal{I}^{2}}\left(y_{1}^{2}+y_{2}^{2}\right)+\varkappa \mathcal{U}(y)+p_{0}=0,
$$

has a solution that determines a smooth axially symmetric equilibrium figure $\mathcal{F}$ satisfying (1.3). We consider the set of surfaces $\Gamma$ given by equations (1.6) in which the functions $\rho$ satisfy inequality (1.7) and are such that the domains $\Omega$ bounded by these surfaces satisfy the conditions

$$
|\Omega|=|\mathcal{F}|, \quad \int_{\Omega} x_{i} d x=0, \quad i=1,2,3 .
$$

These conditions can be expressed in terms of $\rho(y)$ and have the following form (see [1] and also [5]):

$$
\begin{aligned}
& \int_{\mathcal{G}} \varphi(y ; \rho) d S=0 \\
& \int_{\mathcal{G}} \psi_{i}(y ; \rho) d S=0, \quad i=1,2,3,
\end{aligned}
$$

where

$$
\begin{aligned}
\varphi(y ; \rho) & =\rho(y)-\frac{\rho^{2}(y)}{2} \mathcal{H}(y)+\frac{\rho^{3}(y)}{3} \mathcal{K}(y), \\
\psi_{k}(y ; \rho) & =y_{k} \varphi(y ; \rho)+N_{k}(y)\left(\frac{\rho^{2}}{2}-\frac{\rho^{3}}{3} \mathcal{H}(y)+\frac{\rho^{4}}{4} \mathcal{K}(y)\right),
\end{aligned}
$$

and $\mathcal{K}(y)$ is the Gaussian curvature of $\mathcal{G}$. Our basic assumption concerning the functional $R$ is as follows:

there are two positive constants $c_{1}$ and $c_{2}$ such that

$$
c_{1}\left\|r^{2}\right\|_{W_{2}^{1}(\mathcal{G})} \leq \delta_{0}^{2} R[r] \leq c_{2}\|r\|_{W_{2}^{1}(\mathcal{G})}^{2}
$$

for all $r(y)$ satisfying (1.7), (2.3), and (2.4). If $\delta$ is sufficiently small, we can replace conditions (2.3) and (2.4) by

$$
\int_{\mathcal{G}} r(y) d S=0, \quad \int_{\mathcal{G}} y_{k} r(y) d S=0, \quad k=1,2,3 .
$$


The first three terms on the right in (1.4), (1.5) correspond to the potential energy related to the capillary, centrifugal, and gravitational forces that act on the fluid in $\Omega$, and the constant $p_{0}$ is the Lagrange multiplier corresponding to the restriction on the volume of $\Omega$ in (2.2). The other multipliers, related to constraints on the position of the barycenter, vanish. If we multiply $(1.2)$ by $\sum_{i=1}^{3} x_{i} N_{i}$ and integrate the result over $\mathcal{G}$, then, after easy calculations, we obtain an expression for $p_{0}$ :

$$
p_{0}=\frac{2 \sigma|\mathcal{G}|}{3|\mathcal{F}|}-\frac{5}{6|\mathcal{F}|}\left(\omega_{0}^{2} \mathcal{I}+\varkappa \int_{\mathcal{F}} \mathcal{U}(x) d x\right)
$$

In the case of $\sigma=0$, this expression was found in 1 .

The second order variation $\delta_{0}^{2} G[r]$ is defined by the formula

$$
\begin{aligned}
\delta_{0}^{2} G[r]= & \int_{\mathcal{G}}\left[\sigma\left(\left|\nabla_{\mathcal{G}} r\right|^{2}+2 \mathcal{K} r^{2}\right)-\frac{\omega_{0}^{2}}{2}\left(\frac{\partial}{\partial N}\left(y_{1}^{2}+y_{2}^{2}\right)-\left(y_{1}^{2}+y_{2}^{2}\right) \mathcal{H}\right) \eta^{2}+p_{0} \mathcal{H} r^{2}\right] d S \\
& +\varkappa \int_{\mathcal{G}}\left(\mathcal{U} \mathcal{H}-\frac{\partial \mathcal{U}}{\partial N}\right) r^{2} d S-\varkappa \int_{\mathcal{G}} \int_{\mathcal{G}} r(y) r(z) \frac{d S_{y} d S_{z}}{|y-z|}
\end{aligned}
$$

which coincides with

$$
\begin{aligned}
\delta_{0}^{2} G[r]= & \int_{\mathcal{G}}\left[\sigma\left(\left|\nabla_{\mathcal{G}} r\right|^{2}+\left(2 \mathcal{K}-\mathcal{H}^{2}\right) r^{2}\right)-\frac{\omega_{0}^{2}}{2} \frac{\partial}{\partial N}\left(y_{1}^{2}+y_{2}^{2}\right) r^{2}\right] d S \\
& -\varkappa \int_{\mathcal{G}} \frac{\partial \mathcal{U}}{\partial N} r^{2} d S-\varkappa \int_{\mathcal{G}} \int_{\mathcal{G}} r(y) r(z) \frac{d S_{y} d S_{z}}{|y-z|}
\end{aligned}
$$

in view of (1.2); consequently,

$$
\begin{aligned}
\delta_{0}^{2} R[r] & \int_{\mathcal{G}}\left[\sigma\left(\left|\nabla_{\mathcal{G}} r\right|^{2}+2 \mathcal{K} r^{2}\right)-\frac{\omega_{0}^{2}}{2}\left(\frac{\partial}{\partial N}\left(y_{1}^{2}+y_{2}^{2}\right)-\left(y_{1}^{2}+y_{2}^{2}\right) \mathcal{H}\right) r^{2}+p_{0} \mathcal{H} r^{2}\right] d S \\
& +\varkappa \int_{\mathcal{G}}\left(\mathcal{U} \mathcal{H}-\frac{\partial \mathcal{U}}{\partial N}\right) r^{2} d S \\
& -\varkappa \int_{\mathcal{G}} \int_{\mathcal{G}} r(y) r(z) \frac{d S_{y} d S_{z}}{|y-z|}+\frac{\omega_{0}^{2}}{\mathcal{I}}\left(\int_{\mathcal{G}}\left(y_{1}^{2}+y_{2}^{2}\right) r d S\right)^{2} \\
= & \int_{\mathcal{G}}\left[\sigma\left(\left|\nabla_{\mathcal{G}} r\right|^{2}+\left(2 \mathcal{K}-\mathcal{H}^{2}\right) r^{2}\right)-\frac{\omega_{0}^{2}}{2} r^{2} \frac{\partial}{\partial N}\left(y_{1}^{2}+y_{2}^{2}\right)\right] d S-\varkappa \int_{\mathcal{G}} \frac{\partial \mathcal{U}}{\partial N} r^{2} d S \\
& -\varkappa \int_{\mathcal{G}} \int_{\mathcal{G}} r(y) r(z) \frac{d S_{y} d S_{z}}{|y-z|}+\frac{\omega_{0}^{2}}{\mathcal{I}}\left(\int_{\mathcal{G}}\left(y_{1}^{2}+y_{2}^{2}\right) r d S\right)^{2},
\end{aligned}
$$

$\omega_{0}=\beta \mathcal{I}^{-1}$. These formulas were deduced in [1] in the case where $\sigma=0$ and in [7] for $\varkappa=0$. The proof of (2.8) was also given in [5, 6], and in the case where the surfaces $\Gamma=\partial \Omega$ can be represented by an equation of the form $r=R(\theta, \varphi)$ in spherical coordinates, the formula for the second variation of $G$ (upon perturbing $\mathcal{G}$ along the vector $\left.\vec{e}_{r}=\frac{\vec{x}}{|x|}\right)$ was obtained in [4].

We turn to problem (1.1) and introduce some auxiliary constructions. Assume that (1.1) has a classical solution defined for $t \in[0, T], T \leq \infty$. We recall the following 
"conservation laws":

$$
\begin{aligned}
\left|\Omega_{t}\right| & =\left|\Omega_{0}\right|=|\mathcal{F}|, \\
\int_{\Omega_{t}} \vec{v}(x, t) d x & =\int_{\Omega_{0}} \vec{v}_{0}(x) d x=0, \\
\int_{\Omega_{t}}(\vec{x} \times \vec{v}(x, t)) d x & =\int_{\Omega_{0}}\left(\vec{x} \times \vec{v}_{0}(x)\right) d x=\beta \vec{e}_{3}, \\
\int_{\Omega_{t}} \vec{x} d x & =\int_{\Omega_{0}} \vec{x} d x=0 .
\end{aligned}
$$

We put

$$
\begin{aligned}
I(t) & =\int_{\Omega_{t}}\left(x_{1}^{2}+x_{2}^{2}\right) d x, \\
\omega(t) & =\frac{\beta}{i(t)}, \\
\vec{v}^{\prime}(x, t) & =\omega(t)\left(\vec{e}_{3} \times \vec{x}\right) .
\end{aligned}
$$

In accordance with A. Poincaré [2], for every fixed $t, \vec{v}^{\prime}(x, t)$ is the velocity of the points of the "equivalent rigid body" occupying the same domain $\Omega_{t}$ as the fluid and rotating about the axis $x_{3}$ with the angular speed $\omega(t)$. The relative velocity

$$
\vec{v}_{r}(x, t)=\vec{v}(x, t)-\vec{v}^{\prime}(x, t)
$$

satisfies

$$
\int_{\Omega_{t}} \vec{v}_{r} \cdot \vec{v}^{\prime} d x=\omega(t) \int_{\Omega_{t}} \vec{v}_{r} \cdot\left[\vec{e}_{3} \times \vec{x}\right] d x=0
$$

(see [2]). Indeed, by (2.11)-(2.12) we have

$$
\begin{aligned}
& \int_{\Omega_{t}} \vec{v}^{\prime} \cdot\left(\vec{v}-\vec{v}^{\prime}\right) d x \\
& \quad=\omega(t) \int_{\Omega_{t}} \vec{v} \cdot\left(\vec{e}_{3} \times \vec{x}\right) d x-\omega^{2}(t) I(t) \\
& \quad=\omega(t) \int_{\Omega_{t}} \vec{e}_{3} \cdot(\vec{x} \times \vec{v}(x, t)) d x-\omega^{2}(t) I(t)=\omega(t)(\beta-\beta)=0 .
\end{aligned}
$$

Next, we introduce the vector fields

$$
\begin{aligned}
& \vec{v}^{\prime \prime}(x, t)=\sum_{i=1}^{3} \alpha_{i}(t) \vec{\eta}_{i}(x)=\vec{\alpha}(t) \times \vec{x}, \\
& \vec{v}^{\perp}(x, t)=\vec{v}(x, t)-\vec{v}^{\prime \prime}(x, t),
\end{aligned}
$$

where $\vec{\eta}_{i}(x)=\vec{e}_{i} \times \vec{x}, i=1,2,3, \vec{e}_{i}=\left(\delta_{i k}\right)_{k=1,2,3}$, and the $\alpha_{i}(t)$ are determined by the orthogonality conditions

$$
\int_{\Omega_{t}} \vec{v}^{\perp} \cdot \vec{\eta}_{i} d x=0, \quad i=1,2,3 .
$$

It is easily seen that (2.15) is equivalent to the linear algebraic system

$$
\sum_{i=1}^{2} S_{k i}(t) \alpha_{i}(t)=\int_{\Omega_{t}} \vec{v} \cdot \vec{\eta}_{k} d x=\beta \delta_{k 3}, \quad k=1,2,3,
$$

where

$$
S_{k i}(t)=\int_{\Omega_{t}} \vec{\eta}_{k} \cdot \vec{\eta}_{i} \cdot d x=\int_{\Omega_{t}}\left(\delta_{k i}|x|^{2}-x_{i} x_{k}\right) d x .
$$


Thus,

$$
\alpha_{i}(t)=\beta S^{i 3}(t),
$$

where $S^{i k}$ are the elements of the matrix inverse to $\left(S_{i j}\right)_{i, j=1,2,3} \equiv \mathcal{S}$.

Using the identities

$$
\begin{aligned}
-\left(\vec{v}^{\prime} \cdot \nabla\right) \vec{v}^{\prime} & =\frac{1}{2} \nabla\left|\vec{v}^{\prime}\right|^{2}=\frac{\beta^{2}}{2 I^{2}(t)} \nabla\left(x_{1}^{2}+x_{2}^{2}\right), \\
-\left(\vec{v}^{\prime \prime} \cdot \nabla\right) \vec{v}^{\prime \prime} & =\frac{1}{2} \nabla\left|\vec{v}^{\prime \prime}\right|^{2},
\end{aligned}
$$

it is easy to show that $\vec{v}_{r}$ and $\vec{v}^{\perp}$ satisfy the relations

$$
\begin{aligned}
\vec{v}_{r t}+(\vec{v} \cdot \nabla) & \vec{v}_{r}+\left(\vec{v}_{r} \cdot \nabla\right) \vec{v}^{\prime}-\nu \nabla^{2} \vec{v}_{r}+\nabla p_{r} \\
& =-\vec{v}_{t}^{\prime}(x, t)=-\omega_{t}^{\prime}(t) \vec{\eta}_{3}(x), \\
\nabla \cdot \vec{v}_{r} & =0, \quad x \in \Omega_{t}, \quad t>0, \\
\vec{v}_{r}(x, 0) & =\vec{v}_{0}(x)-\vec{v}^{\prime}(x, 0), \\
T\left(\vec{v}_{r}, p_{r}\right) \vec{n} & =\left(\sigma H+\frac{1}{2}\left|\vec{v}^{\prime}\right|^{2}+p_{0}+\varkappa U(x, t)\right) \vec{n}, \\
V_{n} & =\vec{v} \cdot \vec{n}, \quad x \in \Gamma_{t}, \quad t>0,
\end{aligned}
$$

where $\vec{v}=\vec{v}_{r}+\vec{v}^{\prime}, p_{r}=p-\frac{1}{2}\left|\vec{v}^{\prime}\right|^{2}-p_{0}$, and

$$
\begin{aligned}
\vec{v}_{t}^{\perp}+(\vec{v} \cdot \nabla) & \vec{v}^{\perp}+\left(\vec{v}^{\perp} \cdot \nabla\right) \vec{v}^{\prime \prime}-\nu \nabla^{2} \vec{v}^{\perp}+\nabla p^{\perp} \\
& =-\vec{v}_{t}^{\prime \prime}(x, t), \\
\nabla \cdot \vec{v}^{\perp} & =0, \quad x \in \Omega_{t}, \quad t>0, \\
\vec{v}^{\perp}(x, 0) & =\vec{v}_{0}(x)-\vec{v}^{\prime \prime}(x, 0), \\
T\left(\vec{v}^{\perp}, p^{\perp}\right) \vec{n} & =\left(\sigma H+\frac{1}{2}\left|\vec{v}^{\prime \prime}\right|^{2}+p_{0}+\varkappa U(x, t)\right) \vec{n}, \\
V_{n} & =\vec{v} \cdot \vec{n}, \quad x \in \Gamma_{t}, \quad t>0,
\end{aligned}
$$

where $\vec{v}=\vec{v}^{\perp}+\vec{v}^{\prime \prime}, p^{\perp}=p-\frac{1}{2}\left|\vec{v}^{\prime \prime}\right|^{2}-p_{0}$.

Largely, we shall study problem (2.17), and it is often convenient to use the coordinate system that rotates about the axis $x_{3}$ with variable angular speed $\omega(t)$. We rewrite the relations (2.17) in this coordinate system. For this, we make the transformation

$$
\begin{aligned}
x & =\mathcal{Z}(\phi(t)) y, \\
\vec{w}(y, t) & =\mathcal{Z}^{-1}(\phi(t)) \vec{v}_{r}(\mathcal{Z}(\phi(t)) y, t),
\end{aligned}
$$

where

$$
\mathcal{Z}(\lambda)=\left(\begin{array}{ccc}
\cos \lambda & -\sin \lambda & 0 \\
\sin \lambda & \cos \lambda & 0 \\
0 & 0 & 1
\end{array}\right)
$$

and $\phi(t)=\int_{0}^{t} \omega(s) d s$, so that $\phi_{t}^{\prime}(t)=\omega(t)$. We use the relations

$$
\begin{aligned}
\vec{\eta}_{3}(x) & =\left(\mathcal{Z} \vec{e}_{3} \times \mathcal{Z} \vec{y}\right)=\mathcal{Z} \vec{\eta}_{3}(y) \\
\frac{\partial}{\partial t} \vec{v}_{r}(\mathcal{Z} y, t) & =\frac{\partial \vec{v}_{r}}{\partial t}+\left(\frac{d \mathcal{Z}}{d t} y \cdot \nabla_{x}\right) \vec{v}_{r}=\vec{v}_{r t}+\left(\vec{v}^{\prime} \cdot \nabla_{x}\right) \vec{v}_{r} \\
\mathcal{Z}^{-1}(t) \frac{\partial}{\partial t} \vec{v}_{r}(\mathcal{Z} y, t) & =\frac{\partial}{\partial t} \vec{w}(y, t)-\frac{d \mathcal{Z}^{-1}}{d t} \mathcal{Z} \vec{w}=\vec{w}_{t}+\omega(t)\left(\vec{e}_{3} \times \vec{w}\right)
\end{aligned}
$$


and pass to the coordinates $\{y\}$ in the equation

$$
\begin{aligned}
& \mathcal{Z}^{-1}\left(\vec{v}_{r t}+\left(\vec{v}^{\prime} \cdot \nabla\right) \vec{v}_{r}+\left(\vec{v}_{r} \cdot \nabla\right) \vec{v}_{r}+\left(\vec{v}_{r} \cdot \nabla\right) \vec{v}^{\prime}-\nu \nabla^{2} \vec{v}_{r}+\nabla p_{r}\right) \\
& \quad=-\omega^{\prime}(t) \mathcal{Z}^{-1} \vec{\eta}_{3}(x) .
\end{aligned}
$$

After simple calculations, we arrive at the following nonstationary problem with free boundary for the functions $\vec{w}(y, t), s(y, t)=p_{r}(\mathcal{Z} y, t)$ :

$$
\begin{aligned}
\vec{w}_{t}+(\vec{w} \cdot \nabla) \vec{w}+2 \omega(t)\left(\vec{e}_{3} \times \vec{w}\right)-\nu \nabla^{2} \vec{w}+\nabla s & \\
& =-\omega^{\prime}(t) \vec{\eta}_{3}(y), \\
\nabla \cdot \vec{w} & =0, \quad y \in \Omega_{t}^{\prime}=\mathcal{Z}^{-1} \Omega_{t}, \quad t>0, \\
\vec{w}(y, 0) & =\vec{v}_{0}(y)-\vec{v}_{0}^{\prime}(y, 0) \equiv \vec{w}_{0}(y), \\
T(\vec{w}, s) \vec{n} & =\left(\sigma H^{\prime}+\frac{\beta^{2}}{2 I^{\prime 2}(t)}\left(y_{1}^{2}+y_{2}^{2}\right)+p_{0}+\varkappa U^{\prime}(y, t)\right) \vec{n}^{\prime}, \\
V_{n}^{\prime} & =\vec{w} \cdot \vec{n}^{\prime}, \quad y \in \Gamma_{t}^{\prime}=\partial \Omega_{t}^{\prime}, \quad t>0 .
\end{aligned}
$$

Here $\vec{n}^{\prime}$ is the outward unit normal to $\Gamma_{t}^{\prime}, H^{\prime}$ is twice the mean curvature of $\Gamma_{t}^{\prime}, U^{\prime}(y, t)=$ $\int_{\Omega_{t}^{\prime}} \frac{d z}{|y-z|}, V_{n}^{\prime}$ is the velocity of the movement of $\Gamma_{t}^{\prime}$ in the direction of $\vec{n}^{\prime}$, and $I^{\prime}(t)=$ $\int_{\Omega_{t}^{\prime}}\left(y_{1}^{2}+y_{2}^{2}\right) d y$. We deduce the relation $V_{n}^{\prime}=\vec{w} \cdot \vec{n}^{\prime}$ formally. Assume that $\Gamma_{t}$ can be given by an equation of the form $x=X(\xi, t)$, where the point $\xi$ runs over some manifold independent of $t$ (as is the case, e.g., in (1.6)). Then $y=Y(\xi, t)=\mathcal{Z}^{-1} X(\xi, t) \in \Gamma_{t}^{\prime}$, and since $\vec{n}=\mathcal{Z} \vec{n}^{\prime}$, we have

$$
\begin{aligned}
V_{n}^{\prime} & =Y_{t} \cdot \vec{n}^{\prime}=\left(\mathcal{Z}^{-1} X\right)_{t} \cdot \mathcal{Z}^{-1} \vec{n}=\vec{X}_{t} \cdot \vec{n}+\frac{d \mathcal{Z}^{-1}}{d t} \mathcal{Z} Y \cdot \vec{n}^{\prime} \\
& =V_{n}-\omega(t) \vec{\eta}_{3}(y) \cdot \vec{n}^{\prime}=\left(\vec{v}_{r}+\vec{v}^{\prime}\right) \cdot \vec{n}-\omega(t) \vec{\eta}_{3}(y) \cdot \vec{n}=\vec{v}_{r} \cdot \vec{n} \\
& =\vec{w} \cdot \vec{n}^{\prime} .
\end{aligned}
$$

This implies that $I_{t}^{\prime}(t)=\int_{\Omega_{t}^{\prime}} \vec{w} \cdot \nabla\left(y_{1}^{2}+y_{2}^{2}\right) d y$, and

$$
-\omega_{t}^{\prime}(t)=\frac{\beta}{I^{2}(t)} \int_{\Omega_{t}^{\prime}} \vec{w} \cdot \nabla\left(y_{1}^{2}+y_{2}^{2}\right) d y=\frac{2 \beta}{I^{2}(t)} \int_{\Omega_{t}^{\prime}}\left(w_{1} y_{1}+w_{2} y_{2}\right) d y .
$$

Now we formulate the main result of the paper.

Theorem 2.1. Suppose the following:

1) the surface $\Gamma_{0}$ is given by equation (1.6) with $\rho=\rho_{0} \in C^{3+\alpha}(\mathcal{G}), \alpha \in(0,1)$, and conditions (1.7), (1.9), and (1.10) are fulfilled (i.e., $\rho_{0}$ satisfies (2.3) and (2.4)).

2) $\vec{v}_{0} \in C^{2+\alpha}\left(\Omega_{0}\right)$ satisfies the compatibility conditions

$$
\begin{aligned}
\nabla \cdot \vec{v}_{0}(y) & =0, \\
\left(S\left(\vec{v}_{0}\right) \vec{n}_{0}\right)_{\tan } & \equiv S\left(\vec{v}_{0}\right) \vec{n}_{0}-\left.\vec{n}_{0}\left(\vec{n}_{0} \cdot S\left(\vec{v}_{0}\right) \vec{n}_{0}\right)\right|_{y \in \Gamma_{0}}=0,
\end{aligned}
$$

where $\vec{n}_{0}$ is the unit outward normal to $\Gamma_{0}$;

3) the smallness condition

$$
\left\|\vec{w}_{0}\right\|_{L_{2}\left(\Omega_{0}\right)}+\left\|\rho_{0}\right\|_{W_{2}^{1}(\mathcal{G})} \leq \varepsilon \ll 1
$$

is fulfilled.

If $R$ satisfies (2.6) for all $\rho$ subject to (1.7) and (2.7), then problem (2.17) has a unique classical solution defined for all $t \geq 0$, the surface $\Gamma_{t}$ can be given by equation (1.6) with 
$\rho(\cdot, t) \in C^{3+\alpha}(\mathcal{G})$, and

$$
\begin{aligned}
\sup _{t>0} & \left|\vec{v}_{r}(\cdot, t)\right|_{C^{\alpha}\left(\Omega_{t}\right)}+\sup _{t>0}\left|\vec{v}_{r}(\cdot, t)\right|_{C^{2+\alpha}\left(\Omega_{t}\right)}+\sup _{t>0}\left|p_{r}(\cdot, t)\right|_{C^{1+\alpha}\left(\Omega_{t}\right)} \\
& +\sup _{t>0}|\rho(\cdot t)|_{C^{3+\alpha}(\mathcal{G})}+\sup _{t>0}\left|\rho_{t}(\cdot, t)\right|_{C^{2+\alpha}(\mathcal{G})}+\sup _{t>0}\left|\rho_{t t}(\cdot, t)\right|_{C^{\alpha}(\mathcal{G})} \\
\leq & c e^{-b t}\left(\left|\vec{w}_{0}\right|_{C^{2+\alpha}\left(\Omega_{0}\right)}+\left|\rho_{0}\right|_{C^{3+\alpha}(\mathcal{G})}\right), \quad b>0 .
\end{aligned}
$$

The symbols $C^{l}\left(\Omega_{t}\right)$ and $C^{l}(\mathcal{G})$ with nonintegral $l>0$ denote the standard Hölder spaces. Although the smallness condition is imposed on the $L_{2}$-norm of $\vec{w}_{0}$ and the $W_{2}^{1}$ norm of $\rho_{0}$, interpolation inequalities allow us to show that the norms $\left|\vec{w}_{0}\right|_{C^{l}\left(\Omega_{0}\right)}$ and $\left|\rho_{0}\right|_{C^{l+1}\left(\Omega_{0}\right)}$ are also small with arbitrary $l<2+\alpha$; the latter norms are estimated by $c \varepsilon^{\gamma}$ with some $\gamma \in(0,1)$ depending on $l$ (see [6]).

Estimate $(2.25)$ indicates that $\vec{v}(x, t)-\overrightarrow{\mathcal{V}}(x), p(x, t)-\mathcal{P}(x)$ tend exponentially to zero as $t \rightarrow \infty$. This follows from (2.25) and the relations

$$
\begin{aligned}
& \vec{v}(x, t)-\overrightarrow{\mathcal{V}}(x)=\vec{v}_{r}(x, t)+\frac{\beta}{I(t) \mathcal{I}}(\mathcal{I}-I(t)) \vec{\eta}_{3}(x), \\
& p(x, t)-\mathcal{P}(x)=p_{r}(x, t)+\frac{\beta^{2}}{2 I^{2}(t) \mathcal{I}^{2}}\left(\mathcal{I}^{2}-I^{2}(t)\right)\left(x_{1}^{2}+x_{2}^{2}\right),
\end{aligned}
$$

and

$$
\begin{aligned}
I(t)-\mathcal{I}= & \int_{\Omega_{t}}\left(x_{1}^{2}+x_{2}^{2}\right) d x-\int_{\mathcal{F}}\left(y_{1}^{2}+y_{2}^{2}\right) d y \\
= & \int_{0}^{1} d s \int_{\mathcal{G}}\left[\left(y_{1}+s N_{1}(y) \rho(y, t)\right)^{2}+\left(y_{2}+s N_{2} \rho\right)^{2}\right] \\
& \times \rho\left(1-s \rho \mathcal{H}+s^{2} \rho^{2} \mathcal{K}\right) d S_{y}
\end{aligned}
$$

(see [5, §2]).

\section{§3. Solvability of Problem (2.17) on A Finite time interval}

In order to prove Theorem 2.1, it suffices to show that problem (2.17) is solvable locally (in time), and to obtain uniform estimates of the solution. For this, we apply the following theorem on the solvability of the linear problem

$$
\begin{gathered}
\vec{v}_{t}-\nu \nabla^{2} \vec{v}+\nabla p=\vec{f}(\xi, t), \\
\nabla \cdot \vec{v}=g(\xi, t), \quad \xi \in \Omega_{0}, \quad t \in[0, T], T<\infty \\
\vec{v}(\xi, 0)=\vec{v}_{0}(\xi), \\
\Pi_{0} S(\vec{v}) \vec{n}=\vec{b}(\xi, t), \\
\vec{n}_{0} \cdot T(\vec{v}, p) \vec{n}_{0}-\sigma \vec{n}_{0} \cdot \Delta(0) \int_{0}^{t} \vec{v}(\xi, \tau) d \tau=b(\xi, t)+\int_{0}^{t} B(\xi, \tau) d \tau,
\end{gathered}
$$

where $\Pi_{0} \vec{a}=\vec{a}-\vec{n}_{0}\left(\vec{a} \cdot \vec{n}_{0}\right)$ is the projection of the vector $\vec{a}$ to the plane tangent to $\Gamma_{0}$, and $\Delta(0)$ is the Laplace-Beltrami operator on $\Gamma_{0}$.

Theorem 3.1 ([8, 9]). For any $\vec{f}(\cdot, t) \in C^{\alpha}\left(\Omega_{0}\right)$ and any $t \in[0, T], \alpha \in(0,1), g \in$ $C^{1+\alpha}\left(\Gamma_{0}\right), \vec{v}_{0} \in C^{2+\alpha}\left(\Omega_{0}\right), \vec{b} \in C^{1+\alpha \cdot \frac{1+\alpha}{2}}\left(G_{T}\right), G_{T}=\Gamma \times(0, T), b \in C^{1+\alpha}\left(\Gamma_{0}\right)$, and $B \in C^{\alpha}\left(\Gamma_{0}\right)$ satisfying the compatibility conditions

$$
\nabla \cdot \vec{v}_{0}(\xi)=g(\xi, 0), \quad \vec{b}(\xi, t) \cdot \vec{n}_{0}=0, \quad \Pi_{0} S\left(\vec{v}_{0}\right) \vec{n}_{0}=\vec{b}(\xi, 0),
$$

and also the condition

$$
g(\xi, t)=\nabla \cdot \vec{h}(\xi, t), \quad \vec{h}_{t} \in C^{\alpha}\left(\Omega_{0}\right)
$$


problem (3.1) has a unique solution

$$
\vec{v}(\cdot, t) \in C^{2+\alpha}\left(\Omega_{0}\right), \quad p(\cdot, t) \in C^{1+\alpha}\left(\Omega_{0}\right), \quad \vec{v}_{t} \in C^{\alpha}\left(\Omega_{0}\right),
$$

$t \in[0, T]$, and this solution satisfies the inequality

$$
\begin{aligned}
& \sup _{t<T}\left|\vec{v}_{t}(\cdot, t)\right|_{C^{\alpha}\left(\Omega_{0}\right)}+\sup _{t<T}|\vec{v}(\cdot, t)|_{C^{2+\alpha}\left(\Omega_{0}\right)}+\sup _{t<T}|p(\cdot, t)|_{C^{1+\alpha}\left(\Omega_{0}\right)} \\
& \leq c(T)\left(\sup _{t<T}|\vec{f}(\cdot, t)|_{C^{\alpha}\left(\Omega_{0}\right)}+\sup _{t<T}|g(\cdot, t)|_{C^{1+\alpha}\left(\Omega_{0}\right)}\right. \\
&+\sup _{t<T}\left|\vec{h}_{t}(\cdot, t)\right|_{C^{\alpha}\left(\Omega_{0}\right)} \\
&+\left|\vec{v}_{0}\right|_{C^{2+\alpha}\left(\Omega_{0}\right)}+|\vec{b}|_{C^{1+\alpha}, \frac{1+\alpha}{2}\left(G_{T}\right)} \\
&\left.+\sup _{t<T}|b(\cdot, t)|_{C^{1+\alpha}\left(\Gamma_{0}\right)}+\sup _{t<T}|B(\cdot, t)|_{C^{\alpha}\left(\Gamma_{0}\right)}\right) .
\end{aligned}
$$

Here $G_{T}=\Gamma_{0} \times(0, T)$, and $C^{1+\alpha, \frac{1+\alpha}{2}}\left(G_{T}\right)$ is the "parabolic" anisotropic Hölder space.

In order to prove the solvability of problem (2.17), we rewrite it in the rotating coordinate system. Next, in (2.22) we pass to the Lagrangian coordinates $\xi \in \Omega_{0}$ related to the Eulerian coordinates $y \in \Omega_{t}^{\prime}$ by the formula

$$
\vec{y}=\vec{\xi}+\int_{0}^{t} \vec{u}(\xi, \tau) d \tau \equiv \vec{X}(\xi, t),
$$

where $\vec{u}(\xi, t)=\vec{w}(X(\xi, t), t)$. This results in the problem

$$
\begin{gathered}
\vec{u}_{t}-\nu \nabla^{2} \vec{u}+\nabla q(\xi, t)=F_{1}(\vec{u}, q)+F_{1}^{\prime}(\vec{u}), \\
\nabla \cdot \vec{u}=F_{2}(\vec{u}), \quad \xi \in \Omega_{0}, \\
\Pi_{0} S(\vec{u}) \vec{n}_{0}=F_{3}(\vec{u}), \quad \xi \in \Gamma_{0}, \\
\vec{n}_{0} \cdot T(\vec{u}, q) \vec{n}_{0}-\sigma \vec{n}_{0} \cdot \Delta(0) \int_{0}^{t} \vec{u}(\xi, \tau) d \tau \\
=M(\xi)+F_{4}(\vec{u}, q)+F_{5}(\vec{u})+\int_{0}^{t} F_{6}(\vec{u}) d \tau, \\
\vec{u}(\xi, 0)=\vec{w}_{0}(\xi),
\end{gathered}
$$

where $q(\xi, t)=s(X(\xi, t), t)$,

$$
\begin{aligned}
F_{1}(\vec{u}, q)= & \nu\left(\nabla_{u}^{2}-\nabla^{2}\right) \vec{u}+\left(\nabla-\nabla_{u}\right) q, \\
F_{1}^{\prime}(\vec{u})= & -2 \omega(t)\left[\vec{e}_{3} \times \vec{u}\right]-\frac{d \omega(t)}{d t} \vec{\eta}_{3}(X), \\
F_{2}(\vec{u})= & \left(\nabla-\nabla_{u}\right) \cdot \vec{u}=\nabla \cdot \vec{h}(\vec{u}), \quad \vec{h}=\left(I-A^{T}\right) \vec{u}, \\
F_{3}(\vec{u})= & \Pi_{0}\left(\Pi_{0} S(\vec{u}) \vec{n}_{0}-\Pi S_{u}(\vec{u}) \vec{n}\right), \\
F_{4}(\vec{u}, q)= & \left(\vec{n}_{0} \cdot \vec{n}-1\right) q-\nu \vec{n}_{0} \cdot\left(S_{u}(\vec{u}) \vec{n}-S(\vec{u}) \vec{n}_{0}\right), \\
F_{5}(\vec{u})= & \sigma \int_{0}^{t} \vec{n}_{0} \cdot \frac{\partial \Delta(\tau)}{\partial \tau} \vec{\xi} d \tau+\left(\frac{\omega^{2}(t)}{2}\left(X_{1}^{2}+X_{2}^{2}\right)-\frac{\omega^{2}(0)}{2}\left(\xi_{1}^{2}+\xi_{2}^{2}\right)\right) \\
& +\varkappa\left(U(X)-U_{0}(\xi)\right) \vec{n} \cdot \vec{n}_{0}-\left(\sigma H_{0}(\xi)-M(\xi)\right)\left(\vec{n} \cdot \vec{n}_{0}-1\right), \\
F_{6}(\vec{u})= & \sigma \vec{n}_{0} \cdot \frac{\partial \Delta(t)}{\partial t} \int_{0}^{t} \vec{u}(\xi, \tau) d \tau+\sigma \vec{n}_{0} \cdot(\Delta(t)-\Delta(0)) \vec{u}(\xi, t), \\
M(\xi)= & \sigma\left(H_{0}(\xi)-\mathcal{H}(\eta)\right)+\frac{\omega^{2}(0)}{2}\left(\xi_{1}^{2}+\xi_{2}^{2}\right)-\frac{\omega_{0}^{2}}{2}\left(\eta_{1}^{2}+\eta_{2}^{2}\right)+\varkappa\left(U_{0}(\xi)-\mathcal{U}(\eta)\right),
\end{aligned}
$$


and $\eta$ is the point of $\mathcal{G}$ related to $\xi \in \Gamma_{0}$ by $\xi=\eta+N(\eta) \rho_{0}(\eta)$. Here we have used the following notation:

$\nabla_{u}=\mathcal{A} \nabla_{\xi}$ is the transformed gradient $\nabla_{y}$

$\mathcal{A}$ is the matrix of cofactors for the elements

$$
a_{i j}=\delta_{i j}+\int_{0}^{t} \frac{\partial u_{i}}{\partial \xi_{j}} d \tau
$$

of the Jacobi matrix of the transformation (3.3) (the determinant of the latter matrix is equal to 1 );

$\vec{n}$ is the outward normal to $\Gamma_{t}^{\prime}$ at the point $X(\xi, t)$;

$\Pi$ is the projection onto the tangent plane at this point, i.e., $\Pi \vec{a}=\vec{a}-\vec{n}(\vec{a} \cdot \vec{n})$;

$$
S_{u}(\vec{w})=\left(\nabla_{u} \vec{w}\right)+\left(\nabla_{u} \vec{w}\right)^{T}=\left(\sum_{k=1}^{3}\left(A_{k j} \frac{\partial w_{i}}{\partial \xi_{j}}+A_{k i} \frac{\partial w_{j}}{\partial \xi_{i}}\right)\right)_{i, j=1,2,3}
$$

is twice the transformed rate-of-strain tensor $S(\vec{w})$;

$\Delta(t)$ is the Laplace-Beltrami operator on $\Gamma_{t}^{\prime}$;

$\frac{\partial \Delta(t)}{\partial t}$ is the operator obtained from $\Delta(t)$ by differentiating its coefficients with respect to $t$;

$H_{0}(\xi)$ is twice the mean curvature of $\Gamma_{0}$;

$U_{0}(\xi)=\int_{\Omega_{0}} \frac{d \xi^{\prime}}{\left|\xi-\xi^{\prime}\right|}$.

Problems (2.22) and (3.4) are equivalent if $\vec{n} \cdot \vec{n}_{0}>0$.

We prove the following theorem on the solvability of (3.4).

Theorem 3.2. Under the assumptions of Theorem 2.1, problem (3.4) has a unique solution $\vec{u}(\cdot, t) \in C^{2+\alpha}\left(\Omega_{0}\right), q(\cdot, t) \in C^{1+\alpha}\left(\Omega_{0}\right), \vec{u}_{t}(\cdot, t) \in C^{\alpha}\left(\Omega_{0}\right)$ defined on some finite time interval $\left(0, T_{0}\right)$ and satisfying the inequality

$$
\begin{gathered}
\sup _{\tau<t}\left|\vec{u}_{\tau}(\cdot, \tau)\right|_{C^{\alpha}\left(\Omega_{0}\right)}+\sup _{\tau<t}|\vec{u}(\cdot, \tau)|_{C^{2+\alpha}\left(\Omega_{0}\right)}+\sup _{\tau<t}|q(\cdot, t)|_{C^{1+\alpha}\left(\Omega_{0}\right)} \\
\leq c(t)\left(\left|\vec{w}_{0}\right|_{C^{2+\alpha}\left(\Omega_{0}\right)}+\left|\rho_{0}\right|_{C^{3+\alpha}(\mathcal{G})}\right), \quad t \leq T_{0} .
\end{gathered}
$$

The value $T_{0}$ increases unboundedly as the norms on the right in (3.6) tend to zero; $c(t)$ is a monotone nondecreasing function of $t$.

Problem (3.4) differs insignificantly from a similar problem with $\omega=\omega_{0}$ considered in [6. Along with Theorem 3.1, we shall apply some estimates of the expressions $F_{i}$ and $M$; most of them were obtained in 9 .

Lemma 3.1. 1. If $\rho_{0}$ belongs to $C^{3+\alpha}(\mathcal{G})$ and satisfies condition (1.7), then

$$
|M|_{C^{1+\alpha}\left(\Gamma_{0}\right)} \leq c\left|\rho_{0}\right|_{C^{3+\alpha}(\mathcal{G})} .
$$

2. Suppose vector fields $\vec{u}(\xi, t), \vec{u}^{\prime}(\xi, t)$ and functions $q(\xi, t), q^{\prime}(\xi, t)$ are given. Assume that

$$
\begin{aligned}
t\left(\sup _{\tau<t}\left|\vec{u}_{\tau}(\cdot, \tau)\right|_{C^{\alpha}\left(\Omega_{0}\right)}+\sup _{\tau<t}|\vec{u}(\cdot, \tau)|_{C^{2+\alpha}\left(\Omega_{0}\right)}\right) & \leq \delta_{1}, \\
t\left(\sup _{\tau<t}\left|\vec{u}_{\tau}^{\prime}(\cdot, \tau)\right|_{C^{\alpha}\left(\Omega_{0}\right)}+\sup _{\tau<t}\left|\vec{u}^{\prime}(\cdot, \tau)\right|_{C^{2+\alpha}\left(\Omega_{0}\right)}\right) & \leq \delta_{1}, \\
& t \sup _{\tau<t}|q(\cdot, \tau)|_{C^{1+\alpha}\left(\Omega_{0}\right)} \\
\left.t \sup _{\tau<t}\left|q^{\prime}(\cdot, \tau)\right|_{C^{1+\alpha}\left(\Omega_{0}\right)}\right) & \leq \delta_{1},
\end{aligned}
$$

where $\delta_{1}$ is a sufficiently small number. Also, let

$$
\vec{u}-\left.\vec{u}^{\prime}\right|_{t=0}=0 .
$$


Then

$$
\begin{aligned}
\sup _{\tau<t} \mid F_{1} & (\vec{u}, q)-\left.F_{1}\left(\vec{u}^{\prime}, q^{\prime}\right)\right|_{C^{\alpha}\left(\Omega_{0}\right)}+\sup _{\tau<t}\left|F_{2}(\vec{u})-F_{2}\left(\vec{u}^{\prime}\right)\right|_{C^{1+\alpha}\left(\Omega_{0}\right)} \\
& +\left|F_{3}(\vec{u})-F_{3}\left(\vec{u}^{\prime}\right)\right|_{C^{1+\alpha, \frac{1+\alpha}{2}}\left(G_{t}\right)}+\sup _{\tau<t}\left|F_{4}(\vec{u}, q)-F_{4}\left(\vec{u}^{\prime}, q^{\prime}\right)\right|_{C^{1+\alpha}\left(\Gamma_{0}\right)} \\
& +\sup _{\tau<t}\left|F_{6}(\vec{u})-F_{6}\left(\vec{u}^{\prime}\right)\right|_{C^{\alpha}\left(\Gamma_{0}\right)} \\
\leq & c \delta_{1} N_{t}\left(\vec{u}-\vec{u}^{\prime}, q-q^{\prime}\right),
\end{aligned}
$$

where $G_{t}=\Gamma_{0} \times(0, t)$ and

$$
\begin{aligned}
N_{t}(\vec{u}, q)= & \sup _{\tau<t}\left|\vec{u}_{\tau}(\cdot, \tau)\right|_{C^{\alpha}\left(\Omega_{0}\right)} \\
& +\sup _{\tau<t}|\vec{u}(\cdot, t)|_{C^{2+\alpha}\left(\Omega_{0}\right)}+\sup _{\tau<t}|q(\cdot, \tau)|_{C^{1+\alpha}\left(\Omega_{0}\right)} .
\end{aligned}
$$

Moreover, under conditions (3.8) we have

$$
\begin{aligned}
& \sup _{\tau<t}\left|F_{1}^{\prime}(\vec{u})-F_{1}^{\prime}\left(\vec{u}^{\prime}\right)\right|_{C^{\alpha}\left(\Omega_{0}\right)} \leq c \int_{0}^{t}\left|\vec{u}_{\tau}(\cdot, \tau)-\vec{u}_{\tau}^{\prime}(\cdot, \tau)\right|_{C^{\alpha}\left(\Omega_{0}\right)} d \tau, \\
& \sup _{\tau<t}\left|F_{5}(\vec{u})-F_{5}\left(\vec{u}^{\prime}\right)\right|_{C^{\alpha}\left(\Omega_{0}\right)} \leq c \int_{0}^{t}\left|\vec{u}(\cdot, \tau)-\vec{u}^{\prime}(\cdot, \tau)\right|_{C^{2+\alpha}\left(\Omega_{0}\right)} d \tau,
\end{aligned}
$$

and, finally,

$$
\begin{aligned}
\sup _{\tau<t}\left|\frac{\partial}{\partial t}\left(h(\vec{u})-h\left(\vec{u}^{\prime}\right)\right)\right|_{C^{\alpha}\left(\Omega_{0}\right)} \\
\leq c \delta_{1}\left(\sup _{\tau<t}\left|\vec{u}_{\tau}(\cdot, \tau)-\vec{u}_{\tau}\left(\cdot, \tau^{\prime}\right)\right|_{C^{\alpha}\left(\Omega_{0}\right)}\right. \\
\left.\quad \quad+\sup _{\tau<t}\left|\vec{u}(\cdot, \tau)-\vec{u}^{\prime}(\cdot, \tau)\right|_{C^{2+\alpha}\left(\Omega_{0}\right)}\right) \\
\quad+c|\vec{u}(\cdot, 0)|_{C^{\alpha}\left(\Omega_{0}\right)} \sup _{\tau<t}\left|\nabla \vec{u}(\cdot, \tau)-\nabla \vec{u}^{\prime}(\cdot, \tau)\right|_{C^{1+\alpha}\left(\Omega_{0}\right)} .
\end{aligned}
$$

Proof. We start with the proof of (3.7). Consider the difference

$$
\begin{aligned}
& \frac{\omega^{2}(0)}{2}\left(\xi_{1}^{2}+\xi_{2}^{2}\right)-\frac{\omega_{0}^{2}}{2}\left(\eta_{1}^{2}+\eta_{2}^{2}\right) \\
& \quad=\frac{\omega^{2}(0)-\omega_{0}^{2}}{2}\left(\xi_{1}^{2}+\xi_{2}^{2}\right)+\frac{\omega_{0}^{2}}{2}\left(2 \eta_{1} N_{1}+2 \eta_{2} N_{2}\right) \rho_{0}(\eta)+\frac{\omega_{0}^{2}}{2}\left(N_{1}^{2}+N_{2}^{2}\right) \rho_{0}^{2}
\end{aligned}
$$

It is estimated with the help of the relations

$$
\omega^{2}(0)-\omega_{0}^{2}=\frac{\beta^{2}}{I^{2}(0)}-\frac{\beta^{2}}{\mathcal{I}^{2}}
$$

and (2.26), i.e.,

$$
\begin{aligned}
I(0)-\mathcal{I} & =\int_{\Omega_{0}}\left(\xi_{1}^{2}+\xi_{2}^{2}\right) d \xi-\int_{\mathcal{F}}\left(\eta_{1}^{2}+\eta_{2}^{2}\right) d \eta \\
& =\int_{0}^{1} d s \int_{\mathcal{G}}\left[\left(\eta_{1}+s N_{1} \rho_{0}\right)^{2}+\left(\eta_{2}+s N_{2} \rho_{0}\right)^{2}\right] \rho_{0}(\eta)\left(1-s \rho_{0} \mathcal{H}+s^{2} \rho_{0}^{2} \mathcal{K}\right) d S_{\eta} .
\end{aligned}
$$

Obviously, the norm of that difference is dominated by $c\left|\rho_{0}\right|_{C^{3+\alpha}(\mathcal{G})}$. By [6. Proposition 3.1], the expressions $H_{0}(\xi)-\mathcal{H}(\eta)$ and $U_{0}(\xi)-\mathcal{U}(\eta)$ are also estimated by $c\left|\rho_{0}\right|_{C^{3+\alpha}(\mathcal{G})}$, and we arrive at inequality (3.7). 
In order to prove (3.9), we use the fact that the elements $\delta_{i j}-A_{i j}(\xi, t)$ of the matrix $I-\mathcal{A}$ are sums of linear and quadratic functions of the expressions $\int_{0}^{t} \frac{\partial u_{k}(\xi, \tau)}{\partial \xi_{m}} d \tau$, and, consequently, $A_{i j}(\xi, t)-A_{i j}^{\prime}(\xi, t)\left(A_{i j}^{\prime}\right.$ is the cofactor for $\left.\delta_{i j}+\int_{0}^{t} \frac{\partial u_{i}^{\prime}}{\partial \xi_{j}} d \tau\right)$ are sums of terms that contain the differences $\int_{0}^{t}\left(\frac{\partial u_{k}}{\partial \xi_{m}}-\frac{\partial u_{k}^{\prime}}{\partial \xi_{m}}\right) d \tau$ as factors. Thus,

$$
\begin{aligned}
\left|A_{i j}(\cdot, t)-A_{i j}^{\prime}(\cdot, t)\right|_{C^{1+\alpha}\left(\Omega_{0}\right)} & \leq c \int_{0}^{t}\left|\vec{u}(\cdot, \tau)-\vec{u}^{\prime}(\cdot, \tau)\right|_{C^{2+\alpha}\left(\Omega_{0}\right)} d \tau \\
& \leq c t \sup _{\tau<t}\left|\vec{u}(\cdot, \tau)-\vec{u}^{\prime}(\cdot, \tau)\right|_{C^{2+\alpha}\left(\Omega_{0}\right)} .
\end{aligned}
$$

The same can be said about the difference

$$
\vec{n}-\vec{n}^{\prime}=\frac{\mathcal{A} \vec{n}_{0}}{\left|\mathcal{A} \vec{n}_{0}\right|}-\frac{\mathcal{A}^{\prime} \vec{n}_{0}}{\left|\mathcal{A}^{\prime} \vec{n}_{0}\right|}
$$

and about the coefficients of the operator $\Delta(t)-\Delta^{\prime}(t)$, where $\Delta^{\prime}(t)$ is the LaplaceBeltrami operator on $\Gamma_{t}^{\prime}=X^{\prime}\left(\Gamma_{0}, t\right), \vec{X}^{\prime}(\xi, t)=\vec{\xi}+\int_{0}^{t} \vec{u}^{\prime}(\xi, \tau) d \tau$. The definition of $\Delta(t)$ implies that

$$
\Delta(t)=\frac{1}{\sqrt{g}} \sum_{\alpha, \beta=1}^{2} \frac{\partial}{\partial s_{\alpha}} g^{\alpha \beta} \sqrt{g} \frac{\partial}{\partial s_{\beta}}
$$

where $\left(s_{1}, s_{2}\right)$ are local coordinates on $\Gamma_{0}, g=\operatorname{det}\left(g_{\alpha \beta}\right)_{\alpha, \beta=1,2}, g_{\alpha \beta}=\frac{\partial X(\xi(s), t)}{\partial s_{\alpha}} \cdot \frac{\partial X(\xi(s), t)}{\partial s_{\beta}}$, and the $g^{\alpha \beta}$ are the elements of the matrix inverse to $\left(g_{\alpha \beta}\right)_{\alpha, \beta=1,2}$. Detailed calculations can be found in [9, $\S 7]$.

We explain the idea of the proof of inequalities (3.9). Consider, for instance, the expression

$$
\begin{aligned}
F_{2}(\vec{u})-F_{2}\left(\vec{u}^{\prime}\right) & =\left(\nabla_{u}-\nabla_{u^{\prime}}\right) \cdot \vec{u}+\left(\nabla-\nabla_{u^{\prime}}\right) \cdot\left(\vec{u}-\vec{u}^{\prime}\right) \\
& =\left(\mathcal{A}^{\prime}-\mathcal{A}\right) \nabla \cdot \vec{u}+\left(I-\mathcal{A}^{\prime}\right) \nabla \cdot\left(\vec{u}-\vec{u}^{\prime}\right) .
\end{aligned}
$$

Using (3.8) and (3.14), we obtain

$$
\begin{aligned}
\sup _{\tau<t} \mid & F_{2}(\vec{u})-\left.F_{2}\left(\vec{u}^{\prime}\right)\right|_{C^{1+\alpha}\left(\Omega_{0}\right)} \\
\leq & c t\left(\sup _{\tau<t}|\nabla \vec{u}(\cdot, \tau)|_{C^{1+\alpha}\left(\Omega_{0}\right)}+\sup _{\tau<t}\left|\nabla \vec{u}^{\prime}(\cdot, \tau)\right|_{C^{1+\alpha}\left(\Omega_{0}\right)}\right) \\
\quad & \quad \sup _{\tau<t}\left|\vec{u}(\cdot, \tau)-\vec{u}^{\prime}(\cdot, \tau)\right|_{C^{2+\alpha}\left(\Omega_{0}\right)} \\
\leq & c \delta_{1} \sup _{\tau<t}\left|\vec{u}(\cdot, \tau)-\vec{u}^{\prime}(\cdot, \tau)\right|_{C^{2+\alpha}\left(\Omega_{0}\right)} .
\end{aligned}
$$


The other Hölder norms with respect to the spatial variables in (3.9) are estimated in a similar way. Next, let

$$
[f]_{(0, t)}^{(\lambda)}=\sup _{0<h<\tau<t} h^{-\lambda}|f(\tau-h)-f(\tau)|
$$

be the Hölder constant of a function $f(\tau), \tau \in(0, t)$. The norm

$$
\sup _{\Gamma_{0}}\left[F_{3}(\vec{u})-F_{3}\left(\vec{u}^{\prime}\right)\right]_{(0, t)}^{\left(\frac{1+\alpha}{2}\right)}
$$

is estimated with the help of the inequalities

$$
\begin{aligned}
& \sup _{F_{0}}\left|A_{i j}(\xi, \cdot)-\delta_{i j}\right|_{(0, t)}^{\left(\frac{1+\alpha}{2}\right)} \leq c t^{\frac{1-\alpha}{2}} \sup _{\tau<t} \sup _{\Omega_{0}}|\nabla \vec{u}(\xi, \tau)|, \\
& \sup _{\Gamma_{0}}\left[A_{i j}(\xi, \cdot)-A_{i j}^{\prime}(\xi, \cdot)\right]_{(0, t)}^{\left(\frac{1+\alpha}{2}\right)} \\
& \leq c t^{\frac{1-\alpha}{2}}\left(\sup _{\tau<t} \sup _{\Omega_{0}}\left|\nabla \vec{u}(\xi, \tau)-\nabla \vec{u}^{\prime}(\xi, \tau)\right|\right. \\
& +\int_{0}^{t} \sup _{\Omega_{0}}\left|\nabla \vec{u}(\xi, \tau)-\nabla \vec{u}^{\prime}(\xi, \tau)\right| d \tau \\
& \left.\times\left(\sup _{\tau<t} \sup _{\Omega_{0}}|\nabla \vec{u}(\xi, \tau)|+\sup _{\tau<t} \sup _{\Omega_{0}}\left|\nabla \vec{u}^{\prime}(\xi, \tau)\right|\right)\right) .
\end{aligned}
$$

Recalling conditions (3.8) and the interpolation inequality

$$
\begin{aligned}
& \sup _{\Omega_{0}} \mid \nabla \vec{u}(\xi, \tau)-\nabla \vec{u}^{\prime}(\xi, \tau) \mid \\
& \leq \leq\left(t^{\frac{1+\alpha}{2}}\left|\vec{u}-\vec{u}^{\prime}\right|_{C^{2+\alpha}\left(\Omega_{0}\right)}+t^{-\frac{1-\alpha}{2}}\left|\vec{u}-\vec{u}^{\prime}\right|_{C^{\alpha}\left(\Omega_{0}\right)}\right) \\
& \leq c t^{\frac{1+\alpha}{2}}\left(\left|\vec{u}-\vec{u}^{\prime}\right|_{C^{2+\alpha}\left(\Omega_{0}\right)}+\sup _{\tau^{\prime}<\tau}\left|\vec{u}_{\tau^{\prime}}\left(\cdot, \tau^{\prime}\right)-\vec{u}_{\tau^{\prime}}^{\prime}\left(\cdot, \tau^{\prime}\right)\right|_{C^{\alpha}\left(\Omega_{0}\right)}\right)
\end{aligned}
$$

we obtain

$$
\begin{aligned}
& \sup _{\Gamma_{0}}\left[A_{i j}\left(\xi_{j} \cdot\right)-A_{i j}^{\prime}(\xi, \cdot)\right]_{(0, t)}^{\left(\frac{1+\alpha}{2}\right)} \\
& \quad \leq c t\left(\sup _{\tau<t}\left|\vec{u}(\cdot, \tau)-\vec{u}^{\prime}(\cdot, \tau)\right|_{C^{2+\alpha}\left(\Omega_{0}\right)}+\sup _{\tau<t}\left|\vec{u}_{\tau}(\cdot, \tau)-\vec{u}_{\tau}^{\prime}(\cdot, \tau)\right|_{C^{\alpha}\left(\Omega_{0}\right)}\right) .
\end{aligned}
$$

Inequalities similar to (3.17) and (3.18) are also true for the difference (3.15).

Now we represent the difference $F_{3}(\vec{u})-F_{3}\left(\vec{u}^{\prime}\right)$ in the form

$$
\begin{aligned}
F_{3}(\vec{u})-F_{3}\left(\vec{u}^{\prime}\right)=\Pi_{0}\left(\Pi_{0} S\left(\vec{u}-\vec{u}^{\prime}\right) \vec{n}_{0}\right. & \\
& \left.-\Pi S_{u}\left(\vec{u}-\vec{u}^{\prime}\right) \vec{n}\right)-\Pi_{0}\left(\Pi S_{u}\left(\vec{u}^{\prime}\right) \vec{n}-\Pi^{\prime} S_{u^{\prime}}\left(\vec{u}^{\prime}\right) \vec{n}^{\prime}\right)
\end{aligned}
$$


and estimate $\sup _{\Gamma_{0}}\left[F_{3}(\vec{u})-F_{3}\left(\vec{u}^{\prime}\right)\right]_{(0, t)}^{\left(\frac{1+\alpha}{2}\right)}$ by using $(3.17)-(3.18)$. We have

$$
\begin{aligned}
\underset{\Gamma_{0}}{\sup _{\Gamma_{0}}\left[F_{3}(\vec{u})-F_{3}\left(\vec{u}^{\prime}\right)\right]_{(0, t)}^{\left(\frac{1+\alpha}{2}\right)}} & \\
\leq & c \sup _{\Gamma_{0}}\left[\nabla \vec{u}-\nabla \vec{u}^{\prime}\right]_{(0, t)}^{\left(\frac{1+\alpha}{2}\right)} \sup _{\tau<t} \sup _{\Gamma_{0}}\left(|I-\mathcal{A}(\xi, \tau)|+\left|\vec{n}_{0}-\vec{n}\right|\right) \\
& +c \sup _{\tau<t} \sup _{\Gamma_{0}}\left|\nabla \vec{u}-\nabla \vec{u}^{\prime}\right| \sup _{\Gamma_{0}}\left([I-\mathcal{A}]_{(0, t)}^{\left(\frac{1+\alpha}{2}\right)}+\left[\vec{n}_{0}-\vec{n}\right]_{(0, t)}^{\left(\frac{1+\alpha}{2}\right)}\right) \\
& +c \sup _{\Gamma_{0}}\left[\nabla \vec{u}^{\prime}\right]_{(0, t)}^{\left(\frac{1+\alpha}{2}\right)} \sup _{\tau<t} \sup _{\Gamma_{0}}\left(\left|\mathcal{A}(\xi, \tau)-\mathcal{A}^{\prime}(\xi, \tau)\right|+\left|\vec{n}-\vec{n}^{\prime}\right|\right) \\
& +c \sup _{\tau<t} \sup _{\Gamma_{0}}\left|\nabla \vec{u}^{\prime}(\xi, \tau)\right| \sup _{\Gamma_{0}}\left(\left[\mathcal{A}-\mathcal{A}^{\prime}\right]_{(0, t)}^{\left(\frac{1+\alpha}{2}\right)}+\left[\vec{n}-\vec{n}^{\prime}\right]_{(0, t)}^{\left(\frac{1+\alpha}{2}\right)}\right) \\
\leq & c\left(\delta_{1} \sup _{\Gamma_{0}}\left[\nabla \vec{u}-\nabla \vec{u}^{\prime}\right] C_{C^{\frac{1+\alpha}{2}}}(0, t)\right. \\
& \left.\quad+t^{\frac{1-\alpha}{2}} \sup _{\tau<t} \sup _{\Omega_{0}}|\nabla \vec{u}(\xi, \tau)| \sup _{\tau<t} \sup _{\Omega_{0}}\left|\nabla \vec{u}(\xi, \tau)-\nabla \vec{u}^{\prime}(\xi, \tau)\right|\right) \\
& +c t\left(\sup _{\Gamma_{0}}\left[\nabla \vec{u}^{\prime}\right]_{(0, t)}^{\left(\frac{1+\alpha}{2}\right)}+\sup _{\tau<t} \sup _{\Gamma_{0}}\left|\nabla \vec{u}^{\prime}(\xi, \tau)\right|\right) \\
& \times\left(\sup _{\tau<t}\left|\vec{u}(\cdot, \tau)-\vec{u}^{\prime}(\cdot, \tau)\right|_{C^{2+\alpha}\left(\Omega_{0}\right)}+\sup _{\tau<t}\left|\vec{u}_{\tau}(\cdot, \tau)-\vec{u}_{\tau}^{\prime}(\cdot, \tau)\right|_{C^{\alpha}\left(\Omega_{0}\right)}\right) \\
\leq & c \delta_{1}\left(\sup _{\tau<t}\left|\vec{u}(\cdot, \tau)-\vec{u}^{\prime}(\cdot, \tau)\right|_{C^{2+\alpha}\left(\Omega_{0}\right)}+\sup _{\tau<t}\left|\vec{u}_{\tau}(\cdot, \tau)-\vec{u}_{\tau}^{\prime}(\cdot, \tau)\right|_{C^{\alpha}\left(\Omega_{0}\right)}\right),
\end{aligned}
$$

as required.

Inequality (3.11) follows from the relations

$$
\begin{gathered}
F_{1}^{\prime}(\vec{u})-F_{1}^{\prime}\left(\vec{u}^{\prime}\right)=-2\left(\omega(t)-\omega^{\prime}(t)\right)\left[e_{3} \times \vec{u}\right]-2 \omega^{\prime}(t)\left[\vec{e}_{e} \times \vec{u}-\vec{u}^{\prime}\right] \\
-\left(\frac{d \omega(t)}{d t}-\frac{d \omega^{\prime}(t)}{d t}\right) \vec{\eta}_{3}(X)-\frac{d \omega^{\prime}(t)}{d t}\left(\vec{\eta}_{3}(X)-\vec{\eta}_{3}\left(X^{\prime}\right)\right), \\
\omega(t)=\beta / I(t), \quad I(t)=\int_{\Omega_{0}}\left(X_{1}^{2}(\xi, t)+X_{2}^{2}(\xi, t)\right) d \xi \\
\omega^{\prime}(t)=\beta / I^{\prime}(t), \quad I^{\prime}(t)=\int_{\Omega_{0}}\left(X_{1}^{\prime 2}(\xi, t)+X_{2}^{\prime 2}(\xi, t)\right) d \xi \\
\frac{d I(\tau)}{d t}=2 \int_{\Omega_{0}}\left(X_{1} u_{1}+X_{2} u_{2}\right) d \xi \\
\frac{d I^{\prime}(t)}{d t}=2 \int_{\Omega_{0}}\left(X_{1}^{\prime} u_{1}^{\prime}+X_{2}^{\prime} u_{2}^{\prime}\right) d \xi .
\end{gathered}
$$

We have

$$
\begin{aligned}
& \sup _{\tau<t}\left|F_{1}^{\prime}(\vec{u})-F_{1}^{\prime}\left(\vec{u}^{\prime}\right)\right|_{C^{\alpha}\left(\Omega_{0}\right)} \\
& \quad \leq c\left(\sup _{\tau<t}|\vec{u}(\cdot, \tau)|_{C^{\alpha}\left(\Omega_{0}\right)} \int_{0}^{t}\left|\vec{u}-\vec{u}^{\prime}\right|_{C^{\alpha}\left(\Omega_{0}\right)} d \tau+\sup _{\tau<t}\left|\vec{u}(\cdot, \tau)-\vec{u}^{\prime}(\cdot, \tau)\right|_{C^{\alpha}\left(\Omega_{0}\right)}\right) \\
& \quad \leq c \int_{0}^{t}\left|\vec{u}_{\tau}(\cdot, \tau)-\vec{u}_{\tau}^{\prime}\left(\cdot, \tau^{\prime}\right)\right|_{C^{\alpha}\left(\Omega_{0}\right)} d \tau .
\end{aligned}
$$


The difference $F_{5}(\vec{u})-F_{5}\left(\vec{u}^{\prime}\right)$ is estimated in a similar way, with the help of [6] Proposition 3.2 ] and the inequality

$$
\left|\vec{n}_{0} \cdot\left(\frac{\partial \Delta(\tau)}{\partial \tau}-\frac{\partial \Delta^{\prime}(\tau)}{\partial \tau}\right) \vec{\xi}\right|_{C^{1+\alpha}\left(\Gamma_{0}\right)} \leq c\left|\vec{u}(\cdot, \tau)-\vec{u}^{\prime}(\cdot, \tau)\right|_{C^{2+\alpha}\left(\Gamma_{0}\right)}
$$

(the lower terms of the operator $\frac{\partial \Delta(\tau)}{\partial \tau}-\frac{\partial \Delta^{\prime}(\tau)}{\partial \tau}$, which contain the second order derivatives of $\vec{u}-\vec{u}^{\prime}$, disappear because the vector $\vec{n}_{0}$ is orthogonal to the first order derivatives of $\vec{\xi}$ with respect to the tangential variables).

Finally, we consider the difference

$\frac{\partial \vec{h}(\vec{u})}{\partial t}-\frac{\partial \vec{h}\left(\vec{u}^{\prime}\right)}{\partial t}=\frac{\partial\left(\mathcal{A}^{\prime T}-\mathcal{A}^{T}\right)}{\partial t} \vec{u}+\left(\mathcal{A}^{\prime T}-\mathcal{A}^{T}\right) \vec{u}_{t}-\frac{\partial \mathcal{A}^{\prime T}}{\partial t}\left(\vec{u}-\vec{u}^{\prime}\right)+\left(I-\mathcal{A}^{\prime T}\right)\left(\vec{u}_{t}-\vec{u}_{t}^{\prime}\right)$ and write the first term on the right-hand side in the form

$$
\frac{\partial\left(\mathcal{A}^{\prime T}-\mathcal{A}^{T}\right)}{\partial t} \vec{u}=\frac{\partial\left(\mathcal{A}^{\prime T}-\mathcal{A}^{T}\right)}{\partial t}\left(\vec{u}(\xi, 0)+\int_{0}^{t} \vec{u}_{\tau}(\xi, \tau) d \tau\right) .
$$

The derivatives $\frac{\partial A_{i j}}{\partial t}$ are linear combinations of terms that contain $\frac{\partial u_{k}}{\partial \xi_{l}}$ as factors (the same refers to the leading coefficients of the operator $\frac{\partial \Delta(t)}{\partial t}$, and the coefficients of the first order derivatives depend on $\frac{\partial^{2} u_{k}}{\partial \xi_{l} \partial \xi_{m}}$ in a similar way). As a consequence, we have

$$
\begin{aligned}
\sup _{\tau<t} \mid & \frac{\partial h(\vec{u})}{\partial \tau}-\left.\frac{\partial h\left(\vec{u}^{\prime}\right)}{\partial \tau}\right|_{C^{\alpha}\left(\Omega_{0}\right)} \\
\leq & c \delta_{1}\left(\sup _{\tau<t}\left|\vec{u}_{\tau}(\cdot, \tau)-\vec{u}_{\tau}^{\prime}(\cdot, \tau)\right|_{C^{\alpha}\left(\Omega_{0}\right)}+\sup _{\tau<t}\left|\vec{u}(\cdot, \tau)-\vec{u}^{\prime}(\cdot, \tau)\right|_{C^{2+\alpha}\left(\Omega_{0}\right)}\right) \\
& +c|\vec{u}(\cdot, 0)|_{C^{\alpha}\left(\Omega_{0}\right)} \sup _{\tau<t}\left|\nabla \vec{u}(\cdot, \tau)-\nabla \vec{u}^{\prime}(\cdot, \tau)\right|_{C^{1+\alpha}\left(\Omega_{0}\right)},
\end{aligned}
$$

and the last term here does not exceed the quantity

$$
c t^{1 / 2}|\vec{u}(\cdot, 0)|_{C^{\alpha}\left(\Omega_{0}\right)}\left(\sup _{\tau<t}\left|\vec{u}(\cdot, \tau)-\vec{u}^{\prime}(\cdot, \tau)\right|_{C^{2+\alpha}\left(\Omega_{0}\right)}+\sup _{\tau<t}\left|\vec{u}_{\tau}(\cdot, \tau)-\vec{u}^{\prime}(\cdot, \tau)\right|_{C^{\alpha}\left(\Omega_{0}\right)}\right) .
$$

The lemma is proved.

Proof of Theorem 3.2. As in [6], we rewrite problem (3.4) in the form of the equation

$$
(\vec{u}, q)=\phi(\vec{u}, q)+\left(\vec{u}_{1}, q_{1}\right),
$$

where $\left(\vec{u}_{1}, q_{1}\right)$ is a solution of problem (3.1) with $\vec{f}=0, g=0, \vec{v}_{0}=\vec{w}_{0}, \vec{b}=0$, $B=0$, and $b=M(\xi)$, and $\phi(\vec{u}, q) \equiv\left(\vec{u}^{\prime}, q^{\prime}\right)$ is a solution of the same problem with $\vec{f}=F_{1}(\vec{u}, q)+F_{1}^{\prime}(\vec{u}), g=F_{2}(\vec{u}), \vec{v}_{0}=0, \vec{b}=F_{3}(\vec{u}), b=F_{4}(\vec{u}, q)+F_{5}(\vec{u})$, and $B=F_{6}(\vec{u})$. To solve equation (3.19), we apply the successive approximation method:

$$
\left(\vec{u}_{m+1}, q_{m+1}\right)=\phi\left(\vec{u}_{m}, q_{m}\right)+\left(\vec{u}_{1}, q_{1}\right),
$$

where $m=1, \ldots\left(\vec{u}_{1}\right.$ and $q_{1}$ are defined as before $)$. Let

$$
N_{t}(\vec{u}, q)=\sup _{\tau<t}\left|\vec{u}_{t}(\cdot, t)\right|_{C^{\alpha}\left(\Omega_{0}\right)}+\sup _{\tau<t}|\vec{u}(\cdot, t)|_{C^{2+\alpha}\left(\Omega_{0}\right)}+\sup _{\tau<t}|q(\cdot, t)|_{C^{1+\alpha}\left(\Omega_{0}\right)} .
$$

From (3.2) and (3.7) it follows that

$$
N_{t}\left(\vec{u}_{1}, q_{1}\right) \leq c(t)\left(\left|\rho_{0}\right|_{C^{3+\alpha}(\mathcal{G})}+\left|\vec{w}_{0}\right|_{C^{2+\alpha}\left(\Omega_{0}\right)}\right) .
$$

The differences $\left(\vec{u}_{m+1}-\vec{u}_{m}, q_{m+1}-q_{m}\right)$ satisfy the equations

$$
\left(\vec{u}_{m+1}-\vec{u}_{m}, q_{m+1}-q_{m}\right)=\phi\left(\vec{u}_{m}, q_{m}\right)-\phi\left(\vec{u}_{m-1}, q_{m-1}\right),
$$


$m=2,3, \ldots$. By $(3.2)$, we have

$$
\begin{aligned}
N_{t}\left(\vec{u}_{m+1}\right. & \left.-\vec{u}_{m}, q_{m+1}-q_{m}\right) \\
\leq c(t) & \left(\sup _{\tau<t}\left|F_{1}\left(\vec{u}_{m}, q_{m}\right)-F_{1}\left(\vec{u}_{m-1}, q_{m-1}\right)\right|_{C^{\alpha}\left(\Omega_{0}\right)}\right. \\
& \quad+\sup _{\tau<t}\left|F_{1}^{\prime}\left(\vec{u}_{m}\right)-F_{1}^{\prime}\left(\vec{u}_{m-1}\right)\right|_{C^{\alpha}\left(\Omega_{0}\right)}+\sup _{\tau<t}\left|F_{2}\left(\vec{u}_{m}\right)-F_{2}\left(\vec{u}_{m-1}\right)\right|_{C^{1+\alpha}\left(\Omega_{0}\right)} \\
& +\sup _{\tau<t}\left|\frac{\partial h\left(\vec{u}_{m}\right)}{\partial t}-\frac{\partial h\left(\vec{u}_{m-1}\right)}{\partial t}\right|_{C^{\alpha}\left(\Omega_{0}\right)}+\left|F_{3}\left(\vec{u}_{m}\right)-F_{3}\left(\vec{u}_{m-1}\right)\right|_{C^{1+\alpha, \frac{1+\alpha}{2}}}\left(G_{t}\right) \\
& +\sup _{\tau<t}\left|F_{4}\left(\vec{u}_{m}, q_{m}\right)-F_{4}\left(\vec{u}_{m-1}, q_{m-1}\right)\right|_{C^{1+\alpha}\left(\Gamma_{0}\right)} \\
& \left.\quad+\sup _{\tau<t}\left|F_{5}\left(\vec{u}_{m}\right)-F_{5}\left(\vec{u}_{m-1}\right)\right|_{C^{1+\alpha}\left(\Gamma_{0}\right)}+\sup _{\tau<t}\left|F_{6}\left(\vec{u}_{m}\right)-F_{6}\left(\vec{u}_{m-1}\right)\right|_{C^{\alpha}\left(\Gamma_{0}\right)}\right) .
\end{aligned}
$$

Now we use Lemma 3.1. If

$$
t N_{t}\left(\vec{u}_{k}, q_{k}\right) \leq \delta_{1}, \quad k=m, m-1
$$

then, by (3.9)-(3.13),

$$
\begin{aligned}
& N_{t}\left(\vec{u}_{m+1}-\vec{u}_{m}, q_{m+1}-q_{m}\right) \\
& \quad \leq c_{1} \delta_{2} N_{t}\left(\vec{u}_{m}-\vec{u}_{m-1}, q_{m}-q_{m-1}\right) \\
& \quad+c_{2} \int_{0}^{t} N_{\tau}\left(\vec{u}_{m}-\vec{u}_{m-1}, q_{m}-q_{m-1}\right) d \tau, \quad \delta_{2}=\delta_{1}+\left|\vec{w}_{0}\right|_{C^{\alpha}\left(\Omega_{0}\right)} t^{1 / 2},
\end{aligned}
$$

and if (3.21) is true for all $k=1, \ldots, m$, then

$$
\begin{aligned}
& \sum_{k=1}^{m} N_{t}\left(\vec{u}_{k+1}-\vec{u}_{k}, q_{k+1}-q_{k}\right) \\
& \leq c_{1} \delta_{2} \sum_{k=2}^{m} N_{t}\left(\vec{u}_{k}-\vec{u}_{k-1}, q_{k}-q_{k-1}\right) \\
& \quad+c_{2} \int_{0}^{t} \sum_{k=2}^{m} N_{\tau}\left(\vec{u}_{k}-\vec{u}_{k-1}, q_{k}-q_{k-1}\right) d \tau+N_{t}\left(\vec{u}_{2}-\vec{u}_{1}, q_{2}-q_{1}\right) .
\end{aligned}
$$

Now suppose that $\delta_{2}$ is so small that $c_{1} \delta_{2}<1 / 2$ (this is the case for small $\delta_{1}$ and $\varepsilon$ ). Then, applying Gronwall's lemma, from (3.22) we can easily deduce the estimate

$$
\sum_{k=1}^{m} N_{t}\left(\vec{u}_{k+1}-\vec{u}_{k}, q_{k+1}-q_{k}\right) \leq c(t) N_{t}\left(\vec{u}_{2}-\vec{u}_{1}, q_{2}-q_{1}\right) \leq c(t) N_{t}\left(\phi\left(\vec{u}_{1}, q_{1}\right)\right) .
$$

Consequently,

$$
N_{t}\left(\vec{u}_{m+1}, q_{m+1}\right) \leq c(t) \phi\left(\vec{u}_{1}, q_{1}\right)+N_{t}\left(\vec{u}_{1}, q_{1}\right),
$$

and if we require the inequality

$$
T_{0}\left(c\left(T_{0}\right) N_{T_{0}}\left(\phi\left(\vec{u}_{1}, q_{1}\right)+N_{T_{0}}\left(\vec{u}_{1}, q_{1}\right)\right) \leq \delta_{1},\right.
$$

then condition (3.21) will remain true for $k=m+1$. Hence, it will hold for all $k \geq 1$, and (3.23) will be fulfilled for $m=\infty$. The sequence $\left(\vec{u}_{m}, q_{m}\right)$ will converge to a solution of equation (3.19), i.e., of problem (3.4). By using (3.2) and (3.20), it is not difficult to verify that

$$
\phi\left(\vec{u}_{1}, q_{1}\right) \leq c\left(\left|\vec{v}_{0}\right|_{C^{2 \alpha}\left(\Omega_{0}\right)}+\left|\rho_{0}\right|_{C^{3+\alpha}(\mathcal{G})}\right) .
$$

Thus, (3.25) is valid for

$$
T_{0}\left(\left|\vec{v}_{0}\right|_{C^{2+\alpha}\left(\Omega_{0}\right)}+\left|\rho_{0}\right|_{C^{3+\alpha}(\mathcal{G})}\right) \leq \delta_{3} \ll 1 .
$$


This implies that $T_{0} \rightarrow \infty$ as the norms of $\vec{v}_{0}$ and $\rho_{0}$ tend to zero. Estimate (3.4) follows from (3.24), (3.20), and (3.26). The uniqueness of the solution just constructed can be easily proved with the help of (3.2) and (3.9)-(3.13). The theorem is proved.

The solvability of problem (3.4) implies that of (2.22), together with the estimate

$$
\begin{gathered}
\sup _{\tau<t}\left|\vec{w}_{\tau}(\cdot, \tau)\right|_{C^{\alpha}\left(\Omega_{\tau}^{\prime}\right)}+\sup _{\tau<t}\left|\vec{w}_{\tau}(\cdot, \tau)\right|_{C^{2+\alpha}\left(\Omega_{\tau}^{\prime}\right)}+\sup _{\tau<t}|s(\cdot, \tau)|_{C^{1+\alpha}\left(\Omega_{\tau}^{\prime}\right)} \\
\leq c\left(\left|\vec{w}_{0}\right|_{C^{2+\alpha}\left(\Omega_{0}\right)}+\left|\rho_{0}\right|_{C^{1+\alpha}(\mathcal{G})}\right), \quad t \leq T_{0},
\end{gathered}
$$

for its solution. Moreover, if $\varepsilon$ is sufficiently small, then $\Gamma_{t}^{\prime}$ is given by equation (1.6) with $\rho=\rho^{\prime}(y, t)$, and moreover, $\rho^{\prime}(y, 0)=\rho_{0}(y), \rho^{\prime}$ satisfies (1.7), and

$$
\begin{aligned}
& \sup _{\tau<t}\left|\rho^{\prime}(\cdot, \tau)\right|_{C^{3+\alpha}(\mathcal{G})}+\sup _{\tau<t}\left|\rho_{t}^{\prime}(\cdot, \tau)\right|_{C^{1+\alpha}(\mathcal{G})}+\sup _{\tau<t}\left|\rho_{\tau \tau}^{\prime}(\cdot, \tau)\right|_{C^{\alpha}(\mathcal{G})} \\
& \leq c\left(\left|\vec{w}_{0}\right|_{C^{2+\alpha}\left(\Omega_{0}\right)}+\left|\rho_{0}\right|_{C^{1+\alpha}(\mathcal{G})}\right)
\end{aligned}
$$

(see [6. Theorem 3.3]). But then problem (2.17) is also solvable on the interval $\left(0, T_{0}\right)$, and $\Gamma_{t}=\mathcal{Z} \Gamma_{t}^{\prime}$ is determined by equation (1.6) with $\rho=\rho(y, t)=\rho^{\prime}\left(\mathcal{Z}^{-1} y, t\right)$. By (3.27), (3.28), we obtain the estimate

$$
\begin{aligned}
\sup _{\tau<t} \mid & \left.\vec{v}_{\tau}(\cdot, \tau)\right|_{C^{\alpha}\left(\Omega_{\tau}\right)}+\sup _{\tau<t}\left|\vec{v}_{\tau}(\cdot, \tau)\right|_{C^{2+\alpha}\left(\Omega_{\tau}\right)}+\sup _{\tau<t}\left|p_{r}(\cdot, \tau)\right|_{C^{1+\alpha}\left(\Omega_{\tau}\right)} \\
& +\sup _{\tau<t}|q(\cdot, \tau)|_{C^{3+\alpha}(\mathcal{G})}+\sup _{\tau<t}\left|\rho_{\tau}(\cdot, \tau)\right|_{C^{2+\alpha}(\mathcal{G})}+\sup _{\tau<t}\left|\rho_{\tau \tau}(\cdot, \tau)\right|_{C^{\alpha}(\mathcal{G})} \\
\leq & c\left(\left|\vec{w}_{0}\right|_{C^{2+\alpha}\left(\Omega_{0}\right)}+\left|\rho_{0}\right|_{C^{1+\alpha}(\mathcal{G})}\right), \quad t \leq T_{0} .
\end{aligned}
$$

\section{§4. Estimation of the solution of problem $(2.17)$}

Along with Theorem 3.2, we need uniform (in time) estimates for the solution of problem (2.17). One of them is an estimate for the generalized energy. The following auxiliary assertion will help us to deduce it.

Lemma 4.1. Suppose $\Gamma_{t}$ is given by equation (1.6) with a function $\rho(y, t)$ satisfying conditions (1.7) and having first order derivatives with respect to $t$ and second order derivatives with respect to the spatial variables. There exists a solenoidal vector field $\vec{W}(x, t)$ on $\Omega_{t}$ with the following properties:

1)

$$
\vec{W}(x, t) \cdot \vec{n}(x)=m(y ; \rho(y, t)) \varphi(y ; \rho(y, t)), \quad y \in \mathcal{G},
$$

where $x=y+N(y) \rho(y, t) \in \Gamma_{t}, m(y ; \rho)$ is a positive function to be defined later (see (4.6)), and $\varphi(y ; \rho)$ is as in (2.5);

2)

$$
\int_{\Omega_{t}} \vec{W}(x, t) \cdot \vec{\eta}_{i}(x) d x=0, \quad \vec{\eta}_{i}=\vec{e}_{i} \times \vec{x}, \quad i=1,2,3 ;
$$

3) the inequalities

$$
\begin{aligned}
\|\vec{W}(\cdot, t)\|_{W_{2}^{1}\left(\Omega_{t}\right)} & \leq c\|\rho(\cdot, t)\|_{W_{2}^{1 / 2}(\mathcal{G})}, \\
\|\vec{W}(\cdot, t)\|_{L_{2}\left(\Omega_{t}\right)} & \leq c\|\rho(\cdot, t)\|_{L_{2}(\mathcal{G})}, \\
\left\|\vec{W}_{t}(\cdot, t)\right\|_{L_{2}\left(\Omega_{t}\right)} & \leq c\left(\left\|\rho_{t}(\cdot, t)\right\|_{L_{2}(\mathcal{G})}+\|\rho(\cdot, t)\|_{W_{2}^{1 / 2}(\mathcal{G})}\right)
\end{aligned}
$$

are true with constants independent of $t$ (but depending on the maxima of the absolute values of the derivatives of $\rho$ mentioned above). 
Proof. Obviously, there exists a solenoidal vector field $\vec{W}_{0}(y, t), y \in \mathcal{F}$, such that

$$
\begin{aligned}
\vec{W}_{0} \cdot \vec{N} & =\varphi(y ; \rho), \quad y \in \mathcal{G}, \\
\left\|\vec{W}_{0}\right\|_{W_{2}^{1}(\mathcal{F})} & \leq c\|\varphi\|_{W_{2}^{1 / 2}(\mathcal{G})} \leq c\|\rho\|_{W^{1 / 2}(\mathcal{G})}, \\
\left\|\vec{W}_{0}\right\|_{L_{2}(\mathcal{F})} & \leq c\|\varphi\|_{L_{2}(\mathcal{G})} \leq c\|\rho\|_{L_{2}(\mathcal{G})}, \\
\left\|\vec{W}_{0 t}\right\|_{L_{2}(\mathcal{F})} & \leq c\left\|\varphi_{t}\right\|_{L_{2}(\mathcal{G})} \leq c\left\|\rho_{t}\right\|_{L_{2}(\mathcal{G})} .
\end{aligned}
$$

We map $\mathcal{F}$ onto $\Omega_{t}$ by the transformation

$$
x=y+N^{*} \rho^{*} \equiv e_{\rho}(y),
$$

where $N^{*}$ and $\rho^{*}$ are the following extensions of the functions $N$ and $\rho$ from $\mathcal{G}$ to $\mathcal{F}$ : at the points $z=y+N(y) \lambda \in \mathcal{F}\left(y \in \mathcal{G}, \lambda<\lambda_{0}\right)$ close to $\mathcal{G}$, we put

$$
N^{*}(z)=N(y), \quad \rho^{*}(z)=\rho(y) \psi(\lambda),
$$

where $\psi(\lambda)$ is a smooth cut-off function equal to zero for $|\lambda| \geq \frac{3 \lambda_{0}}{4}$ and to one for $|\lambda|<\frac{\lambda_{0}}{2}$; outside the $\lambda_{0}$-neighborhood of $\mathcal{G}$, we set $\rho=0$ and extend $N^{*}$ in an arbitrary way. Clearly, for such an extension we have

$$
\left.\frac{\partial N^{*}}{\partial N}\right|_{\mathcal{G}}=0,\left.\quad \frac{\partial \rho^{*}}{\partial N}\right|_{\mathcal{G}}=0 .
$$

Let $\mathcal{L}_{\rho}(y, \rho)=\left(\frac{\partial e_{\rho}(y)}{\partial y}\right)=\left(\delta_{i j}+\frac{\partial N_{i}^{*} \rho^{*}}{\partial y_{j}}\right)_{i, j=1,2,3}$ be the Jacobi matrix of the transformation (4.5), let $L_{\rho}=\operatorname{det} \mathcal{L}_{\rho}$, and let $\widehat{\mathcal{L}}_{\rho}=L_{\rho} \mathcal{L}_{\rho}^{-1}=\left(\hat{L}_{i j}(y ; \rho)\right)_{i, j=1,2,3}$ be the matrix reciprocal to $\mathcal{L}_{\rho}$ (for $\delta$ sufficiently small the transformation (4.4) is obviously invertible and the matrix $\mathcal{L}_{\rho}^{-1}$ is well defined). Let

$$
\vec{W}^{\prime}(x, t)=\left.\frac{1}{L_{\rho}(y)} \mathcal{L}_{\rho} \vec{W}_{0}(y, t)\right|_{y=e_{\rho}^{-1}(x)}
$$

(i.e., $\vec{W}_{0}=\widehat{\mathcal{L}}_{\rho} \vec{W}^{\prime}$ ). The Piola identity

$$
\sum_{j=1}^{3} \frac{\partial}{\partial y_{j}} \hat{L}_{j k}=0, \quad k=1,2,3,
$$

implies that $\nabla_{x} \cdot \vec{W}^{\prime}(x, t)=0$; moreover, since

$$
\vec{n}(x)=\frac{1}{\left|\widehat{\mathcal{L}}_{\rho}^{T} \vec{N}\right|} \widehat{\mathcal{L}}^{T} \vec{N}(y),
$$

we see that condition (4.1) is fulfilled,

$$
\vec{W}^{\prime} \cdot \vec{n}=\left.\frac{\varphi(y, \rho)}{\left|\widehat{\mathcal{L}}^{T} \vec{N}(y)\right|}\right|_{y=e_{\rho}^{-1}(x)}, \quad x \in \Gamma_{t},
$$

with $m(y ; \rho)=\left|\widehat{\mathcal{L}}_{\rho}^{T} N\right|^{-1}$. Obviously,

$$
\begin{gathered}
\left\|\vec{W}^{\prime}(\cdot, t)\right\|_{W_{2}^{1}\left(\Omega_{t}\right)} \leq c\left\|\vec{W}_{0}(\cdot, t)\right\|_{W_{2}^{1}(\mathcal{F})} \leq c\|\rho\|_{W_{2}^{1 / 2}(\mathcal{G})}, \\
\left\|\vec{W}^{\prime}(\cdot, t)\right\|_{L_{2}\left(\Omega_{t}\right)} \leq c\left\|\vec{W}_{0}(\cdot, t)\right\|_{L_{2}(\mathcal{F})} \leq c\|\rho\|_{L_{2}(\mathcal{G})}
\end{gathered}
$$

moreover, the relation

$$
\frac{\partial \vec{W}^{\prime}(x, t)}{\partial t}=\frac{\partial \vec{W}^{\prime}\left(e_{\rho}(y), t\right)}{\partial t}-\sum_{k=1}^{3} \frac{\partial \vec{W}^{\prime}}{\partial x_{k}} N_{k}^{*} \frac{\partial \rho^{*}}{\partial t}
$$


and (4.4) imply

$$
\begin{aligned}
\left\|\frac{\partial \vec{W}^{\prime}(\cdot, t)}{\partial t}\right\|_{L_{2}\left(\Omega_{t}\right)} & \leq c\left(\left\|\vec{W}_{0}(\cdot, t)\right\|_{W_{2}^{1}(\Omega)}+\left\|\vec{W}_{0 t}\right\|_{L_{2}\left(\Omega_{t}\right)}\right) \\
& \leq c\left(\left\|\rho_{t}(\cdot, t)\right\|_{L_{2}(\mathcal{G})}+\|\rho\|_{W_{2}^{1 / 2}(\mathcal{G})}\right)
\end{aligned}
$$

Finally, we set

$$
\vec{W}(x, t)=\vec{W}^{\prime}(x, t)-\sum_{i=1}^{3} c_{i}(t) \operatorname{curl} \vec{e}_{i} \mathcal{A}(x),
$$

where $\mathcal{A}(x)$ is a $C_{0}^{\infty}\left(\Omega_{t}\right)$-function with $\int_{\Omega_{t}} \mathcal{A}(x) d x=\frac{1}{2}$, and

$$
c_{i}(t)=\int_{\Omega_{t}} \vec{W}^{\prime}(x, t) \cdot \vec{\eta}_{i}(x) d x .
$$

Since $\operatorname{curl} \vec{\eta}_{j}(x)=2 \vec{e}_{j}$, we have

$$
\int_{\Omega_{t}} \vec{W}(x, t) \cdot \vec{\eta}_{j}(x) d x=c_{j}-\sum_{i=1}^{3} c_{i} \vec{e}_{i} \cdot \int_{\Omega_{t}} \mathcal{A}(x) \operatorname{curl} \vec{\eta}_{j}(x) d x=0 ;
$$

estimates (4.3) follow from (4.7)-(4.9). The lemma is proved.

Theorem 4.1. Assume that problem (2.17) has a classical solution defined for $t \in[0, T]$, $T \leq \infty$, and that $\Omega_{t}$ satisfies the assumptions of Lemma 4.1. If condition (2.6) is fulfilled, then there exists a function $E(t)$ satisfying the inequalities

(4.11) $c_{1}\left(\left\|\vec{v}_{r}(\cdot, t)\right\|_{L_{2}\left(\Omega_{t}\right)}^{2}+\|\rho(\cdot, t)\|_{W_{2}^{1}(\mathcal{G})}^{2}\right) \leq E(t) \leq c_{2}\left(\left\|\vec{v}_{r}(\cdot, t)\right\|_{L_{2}\left(\Omega_{t}\right)}^{2}+\|\rho(\cdot, t)\|_{W_{2}^{1}(\mathcal{G})}^{2}\right)$,

$$
E(t) \leq e^{-b t} E(0), \quad t \in[0, T],
$$

with constants $c_{1}, c_{2}, b>0$ independent of $t$.

Proof. First, we prove the energy inequality for the relative velocity $\vec{v}_{r}$. For this, we multiply the first equation in (1.1) by $\vec{v}$ and integrate over $\Omega_{t}$, taking into account (2.13) and also the relations

$$
\begin{aligned}
\frac{\partial}{\partial t} \int_{\Omega_{t}} U d x & =\int_{\Omega_{t}}\left(U_{t}+(\vec{v} \cdot \nabla) U\right) d x \\
& =\int_{\Omega_{t}} d x \int_{\Omega_{t}}\left(\vec{v}(y, t) \cdot \nabla_{y}\right) \frac{1}{|x-y|} d y+\int_{\Omega_{t}}\left(\vec{v} \cdot \nabla_{x}\right) U(x, t) d x \\
& =2 \int_{\Omega_{t}}\left(\vec{v} \cdot \nabla_{x}\right) U(x, t) d x=2 \int_{\Gamma_{t}} U \vec{v} \cdot \vec{n} d S \\
\frac{d\left|\Gamma_{t}\right|}{d t} & =\frac{d}{d t} \int_{\mathcal{G}}\left|\widehat{\mathcal{L}}^{T} \vec{N}(y)\right| d S_{y} \\
& =\int_{\mathcal{G}} \sum_{i, k, m=1}^{3} N_{k} \widehat{L}_{k i} N_{m} \frac{\partial \widehat{L}_{m i}}{\partial t} \frac{d S_{y}}{\left|\widehat{\mathcal{L}}^{T} N\right|}=\sum_{i, m=1}^{3} \int_{\mathcal{G}} n_{i} N_{m} \frac{\partial \widehat{L}_{m i}}{\partial t} d S_{y} \\
& =\sum_{i, k, m, s=1}^{3} \int_{\mathcal{G}}\left(-n_{i} N_{m} \widehat{L}_{m k} \frac{\partial^{2} N_{k} \rho}{\partial y_{s} \partial t} l^{s i}+n_{i} N_{m} \widehat{L}_{m i} \frac{\partial^{2} N_{k} \rho}{\partial y_{s} \partial t} l^{k s}\right) d S_{y} \\
& =\int_{\Gamma_{t}}\left(-\sum_{i, k=1}^{3} n_{i} n_{k} \frac{\partial^{2} N_{k} \rho}{\partial x_{i} \partial t}+\sum_{k=1}^{3} \frac{\partial^{2} N_{k} \rho}{\partial x_{k} \partial t}\right) d S_{x}=\sum_{k=1}^{3} \int_{\Gamma_{t}} \partial_{k}\left(N_{k} \rho_{t}\right) d S
\end{aligned}
$$




$$
=\sum_{k=1}^{3} \int_{\Gamma_{t}}(\vec{n} \cdot \vec{N}) H \rho_{t} d S=-\int_{\Gamma_{t}} V_{n} H d S=-\int_{\Gamma_{t}} \vec{v} \cdot \vec{n} H d S,
$$

where the $l^{s i}=\frac{\partial y^{s}}{\partial x_{i}}$ are the elements of the matrix $\mathcal{L}_{\rho}^{-1}$, and $\partial_{k}=\frac{\partial}{\partial x_{k}}-n_{k} \frac{\partial}{\partial n}=\vec{e}_{k} \cdot \nabla_{\Gamma_{t}}$ (compare with [5, §2]). As a result, we obtain

$$
\frac{1}{2} \frac{d}{d t}\|\vec{v}\|_{L_{2}\left(\Omega_{t}\right)}^{2}+\frac{\nu}{2}\|S(\vec{v})\|_{L_{2}\left(\Omega_{t}\right)}^{2}=-\sigma \frac{d}{d t}\left|\Gamma_{t}\right|+\frac{\varkappa}{2} \frac{d}{d t} \int_{\Omega_{t}} U(x, t) d x .
$$

Since $S(\vec{v})=S\left(\vec{v}_{r}\right)$ and

$$
\|\vec{v}\|_{L_{2}\left(\Omega_{t}\right)}^{2}=\left\|\vec{v}_{r}\right\|_{L_{2}\left(\Omega_{t}\right)}^{2}+\left\|\vec{v}^{\prime}\right\|_{L_{2}\left(\Omega_{t}\right)}^{2}=\left\|\vec{v}_{r}\right\|_{L_{2}\left(\Omega_{t}\right)}^{2}+\frac{\beta^{2}}{I(t)}
$$

we can rewrite (4.13) in the form

$$
\frac{d}{d t}\left(\frac{1}{2}\left\|\vec{v}_{r}(\cdot, t)\right\|_{L_{2}\left(\Omega_{t}\right)}^{2}+R[\rho]-R[0]\right)+\frac{\nu}{2}\left\|S\left(\vec{v}_{r}\right)\right\|_{L_{2}\left(\Omega_{t}\right)}^{2}=0
$$

where $R[0]$ is the value of the functional (1.5) for $\rho=0$ (i.e., for $\Omega=\mathcal{F}$ ). This identity can also be obtained by multiplying the first equation in (2.17) by $\vec{v}_{r}$ and integrating over $\Omega_{t}$, or by multiplying the first equation in (2.22) by $\vec{w}$ and then integrating over $\Omega_{t}^{\prime}$. The difference

$$
\begin{aligned}
R[\rho]-R[0] & =\int_{0}^{1} \frac{d}{d s} R[s \rho] d s=\int_{0}^{1}\left(\frac{d}{d s} R[s \rho]-\frac{d}{d s^{\prime}} R\left[s^{\prime} \rho\right]_{s^{\prime}=0}\right) d s \\
& =\int_{0}^{1} d s \int_{0}^{s} \frac{d^{2}}{d \lambda^{2}} R[\lambda \rho] d \lambda=\int_{0}^{1}(1-\lambda) \frac{d^{2}}{d \lambda^{2}} R[\lambda \rho] d \lambda \\
& =\frac{1}{2} \delta_{0}^{2} R[\rho]+\int_{0}^{1}(1-\lambda)\left(\frac{d^{2}}{d \lambda^{2}} R[\lambda \rho]-\left.\frac{d^{2}}{d \lambda^{2}} R[\lambda \rho]\right|_{\lambda=0}\right) d \lambda
\end{aligned}
$$

can be estimated from above and from below by $c\|\rho\|_{W_{2}^{1}(\mathcal{G})}^{2}$ if $\delta$ is sufficiently small. However, (4.14) does not imply (4.12) yet. As the second step, we multiply the first equation in (2.18) by the vector $\vec{W}$ constructed in Lemma 4.1 and integrate over $\Omega_{t}$. Using the orthogonality condition (2.15), we obtain

$$
\begin{aligned}
\frac{d}{d t} \int_{\Omega_{t}} & \vec{v}^{\perp} \cdot \vec{W} d x-\int_{\Omega_{t}} \vec{v}^{\perp} \cdot\left(\vec{W}_{t}+(\vec{v} \cdot \nabla) \vec{W}\right) d x \\
& +\int_{\Omega_{t}}\left(\vec{v}^{\perp} \cdot \nabla\right) \vec{v}^{\prime \prime} \cdot \vec{W} d x+\frac{\nu}{2} \int_{\Omega_{t}} S\left(\vec{v}^{\perp}\right): S(\vec{W}) d x \\
= & \int_{\Gamma_{t}}\left(\sigma H+\frac{1}{2}\left|\vec{v}^{\prime \prime}\right|^{2}+p_{0}+\varkappa U(x, t)\right) \vec{W} \cdot \vec{n} d S_{x} \\
= & \int_{\Gamma_{t}}\left(\sigma H(x)+\frac{1}{2}\left|\vec{v}^{\prime \prime}(x)\right|^{2}+p_{0}+\varkappa \mathcal{U}(x, t)\right) \frac{\varphi(y, \rho)}{\left|\widehat{\mathcal{L}}^{T} \vec{N}\right|} d S_{x}
\end{aligned}
$$

where $x=y+N \rho(y, t)$. Invoking equation (2.1), we rewrite the last integral in the form

$$
\int_{\mathcal{G}}\left[\sigma(H(x)-\mathcal{H}(y))+\frac{1}{2}\left(\left|\vec{v}^{\prime \prime}(x)\right|^{2}-\omega_{0}^{2}\left(y_{1}^{2}+y_{2}^{2}\right)\right)+\varkappa(U(x, t)-\mathcal{U}(y))\right] \varphi d S_{y} \equiv I_{\mathcal{G}}
$$

and then we add (4.14) to (4.15) multiplied by small $\gamma>0$. This yields

$$
\frac{d}{d t} E(t)+E_{1}(t)=0,
$$


where

$$
\begin{aligned}
E(t)= & \frac{1}{2}\left\|\vec{v}_{r}(\cdot, t)\right\|_{L_{2}\left(\Omega_{t}\right)}^{2}+(R[\rho]-R[0])+\gamma \int_{\Omega_{t}} \vec{v}^{\perp} \cdot \vec{W} d x \\
E_{1}(t)= & \frac{\nu}{2}\left\|S\left(\vec{v}^{\perp}\right)\right\|_{L_{2}(\Omega)}^{2}+\frac{\gamma \nu}{2} \int_{\Omega} S\left(\vec{v}^{\perp}\right): S(\vec{W}) d x \\
& +\gamma \int_{\Omega_{t}}\left(\vec{v}^{\perp} \cdot \nabla\right) \vec{v}^{\prime \prime} \cdot \vec{W} d x-\gamma \int_{\Omega_{t}} \vec{v}^{\perp} \cdot\left(\vec{W}_{t}+(\vec{v} \cdot \nabla) \vec{W}\right) d x-\gamma I_{\mathcal{G}}
\end{aligned}
$$

because $S\left(\vec{v}_{r}\right)=S\left(\vec{v}^{\perp}\right)$. By using (2.6) and (4.3), it is not difficult to prove estimate (4.11) if the number $\gamma>0$ is sufficiently small. To prove (4.12), it suffices to obtain the inequality

$$
E_{1}(t) \geq b E(t)
$$

with some constant $b>0$. Consider the integral $-I_{\mathcal{G}}$, which contains one of the main positive terms in $E_{1}(t)$. We rewrite it as the sum of four terms:

$$
\begin{aligned}
I_{\mathcal{G}}= & I^{(1)}+I^{(2)}+I^{(3)}+I^{(4)}, \\
I^{(1)}= & \int_{\mathcal{G}}\left[\sigma(H(x)-\mathcal{H}(y))+\frac{\omega_{0}^{2}}{2}\left(x_{1}^{2}+x_{2}^{2}\right)-\frac{\omega_{0}^{2}}{2}\left(y_{1}^{2}+y_{2}^{2}\right)+\varkappa(U(x)-\mathcal{U}(y))\right] \rho d S_{y}, \\
I^{(2)}= & \int_{\mathcal{G}}\left(\frac{\omega^{2}(t)}{2}-\frac{\omega_{0}^{2}}{2}\right)\left(x_{1}^{2}+x_{2}^{2}\right) \rho(y) d S_{y}, \\
I^{(3)}= & \frac{1}{2} \int_{\mathcal{G}}\left(\left|\vec{v}^{\prime \prime}(x, t)\right|^{2}-\left|\vec{v}^{\prime}(x, t)\right|\right)^{2} \rho d S_{y}, \\
I^{(4)}= & \int_{\mathcal{G}}\left[\sigma(H(x)-\mathcal{H}(y))+\frac{1}{2}\left(\left|\vec{v}^{\prime \prime}(x)\right|^{2}-\omega_{0}^{2}\left(y_{1}^{2}+y_{2}^{2}\right)\right)+\varkappa(U(x)-\mathcal{U}(y))\right] \\
& \times(\varphi(y, \rho)-\rho) d S_{y}, \quad x=e_{\rho}(y) .
\end{aligned}
$$

In [5, 6] it was shown that

$$
-I^{(1)}=\delta_{0}^{2} G[\rho]+K_{1},
$$

where $\delta_{0}^{2} G$ is as in (2.8), and $K_{1}$ satisfies

$$
\left|K_{1}\right| \leq c \delta\|\rho\|_{W_{2}^{1}(\mathcal{G})}^{2} .
$$

Now we turn to the integral $I^{(2)}$. Since

$$
\frac{\omega^{2}(t)}{2}-\frac{\omega_{0}^{2}}{2}=\frac{\beta^{2}}{2}\left(\frac{1}{I^{2}(t)}-\frac{1}{\mathcal{I}^{2}}\right)=-\frac{\beta^{2}}{2}(I(t)-\mathcal{I})\left(\frac{1}{I(t) \mathcal{I}^{2}}+\frac{1}{\mathcal{I} I^{2}(t)}\right),
$$

and the leading linear (with respect to $\rho$ ) part of the difference

$$
I(t)-\mathcal{I}=\int_{\Omega_{t}}\left(x_{1}^{2}+x_{2}^{2}\right) d x-\int_{\mathcal{F}}\left(y_{1}^{2}+y_{2}^{2}\right) d y
$$

is equal to

$$
\delta_{0} I=\int_{\mathcal{G}}\left(y_{1}^{2}+y_{2}^{2}\right) \rho(y, t) d S_{y}
$$

(this follows from (2.26)), we have

$$
-I^{(2)}=\frac{\beta^{2}}{\mathcal{I}^{3}}\left(\delta_{0} I\right)^{2}+K_{2},
$$


where $K_{2}$ also satisfies (4.19). Consider the integrand of $-I^{(3)}$ :

$$
\begin{aligned}
\left|\vec{v}^{\prime}(x)\right|^{2}-\left|\vec{v}^{\prime \prime}(x)\right|^{2}= & \omega^{2}(t)\left(x_{1}^{2}+x_{2}^{2}\right)-\left(\alpha_{2} x_{3}-\alpha_{3} x_{2}\right)^{2} \\
& -\left(\alpha_{3} x_{1}-\alpha_{1} x_{3}\right)^{2}-\left(\alpha_{1} x_{2}-\alpha_{2} x_{1}\right)^{2} \\
= & \left(\omega^{2}(t)-\alpha_{3}^{2}\right)\left(x_{1}^{2}+x_{2}^{2}\right)-\left(\alpha_{1}^{2}+\alpha_{2}^{2}\right) x_{3}^{2} \\
& +2\left(\alpha_{1} \alpha_{2} x_{1} x_{2}+\alpha_{2} \alpha_{3} x_{2} x_{3}+\alpha_{3} \alpha_{1} x_{1} x_{3}\right) .
\end{aligned}
$$

We use formulas (2.16) for $\alpha_{j}$, and the relations

$$
\begin{aligned}
S^{13} & =\frac{S_{12} S_{23}-S_{22} S_{13}}{\operatorname{det} \mathcal{S}}, \\
S^{23} & =\frac{S_{21} S_{13}-S_{11} S_{23}}{\operatorname{det} \mathcal{S}}, \\
\omega(t)-\alpha_{3} & =\beta\left(\frac{1}{S_{33}}-S^{33}\right)=\frac{\beta}{S_{33}} \sum_{j=1}^{2} S_{3 j} S^{j 3} .
\end{aligned}
$$

Since $\int_{\mathcal{F}} y_{j} y_{3} d y=0, j=1,2$, and $\delta_{0} S_{j 3}=-\int_{\mathcal{G}} y_{j} y_{3} \rho d S_{y}$ (see [5, §2]), we have

$$
S_{j 3}=-\int_{\mathcal{G}} y_{j} y_{3} \rho(y, t) d S_{y}+S_{j 3}^{\prime}, \quad\left|S_{j 3}^{\prime}\right|<c\|\rho\|_{L_{2}(\mathcal{G})}^{2} .
$$

Moreover,

$$
\left|\omega^{2}(t)-\alpha_{3}^{2}\right|+\left|\alpha_{1}^{2}+\alpha_{2}^{2}\right|+\left|\alpha_{1} \alpha_{2}\right| \leq c\|\rho\|_{L_{2}(\mathcal{G})}^{2} .
$$

Next, by (2.16) and (4.20), we have

$$
\int_{\mathcal{G}}\left(\alpha_{1} \alpha_{3} x_{1} x_{3}+\alpha_{2} \alpha_{3} x_{2} x_{3}\right) \rho(y, t) d S_{y}=\beta \alpha_{3} \frac{S_{22} S_{13}^{2}+S_{11} S_{23}^{2}-2 S_{12} S_{13} S_{23}}{\operatorname{det} \mathcal{S}}+K_{3}
$$

where $K_{3}$ satisfies (4.19). Since $\left|S_{12}\right| \leq \sqrt{S_{11} S_{12}}$, the first term on the right in (4.22) is nonnegative, whence

$$
-I^{(3)} \geq K_{4}, \quad\left|K_{4}\right| \leq c \delta\|\rho\|_{W_{2}^{1}(\mathcal{G})}^{2} .
$$

Finally, expressing the difference $H-\mathcal{H}$ in terms of $\rho$ by the formula

$$
\begin{aligned}
H(x)-\mathcal{H}(y) & =\vec{n} \cdot \Delta_{\Gamma_{t}} \vec{x}-\vec{N} \cdot \Delta_{\mathcal{G}} \vec{y} \\
& =\vec{n} \cdot \Delta_{\Gamma_{t}}(\vec{N} \rho)+(\vec{n}-\vec{N}) \cdot \Delta_{\Gamma_{t}} \vec{y}+\vec{N} \cdot\left(\Delta_{\Gamma_{t}}-\Delta_{\mathcal{G}}\right) \vec{y}
\end{aligned}
$$

and integrating by parts, we easily verify that

$$
\left|I^{(4)}\right| \leq c \delta\|\rho\|_{W_{2}^{1}(\mathcal{G})} .
$$

Thus,

$$
-I_{\mathcal{G}} \geq \delta_{0}^{2} R[\rho]+K
$$

where $K$ satisfies (4.19).

Now we consider the volume integrals in (4.17). By (4.3),

$$
\left|\int_{\Omega_{t}} \vec{v}^{\perp} \cdot\left(\vec{W}_{t}+(\vec{v} \cdot \nabla) \vec{W}\right) d x\right| \leq c\left\|\vec{v}^{\perp}\right\|_{L_{2}\left(\Omega_{t}\right)}\left(\left\|\rho_{t}\right\|_{L_{2}(\mathcal{G})}+\|\rho\|_{W_{2}^{1 / 2}(\mathcal{G})}\right) .
$$


In order to estimate $\rho_{t}$, we use the kinematic boundary condition $V_{n}=\vec{v} \cdot \vec{n}$, which can be written equivalently in the form

$$
\begin{aligned}
\rho_{t}(y, t)= & \frac{\vec{v}(x, t) \cdot \vec{n}(x)}{\vec{n}(x) \cdot \vec{N}(y)} \\
= & \frac{1}{\vec{n} \cdot \vec{N}}\left(\vec{v}^{\perp} \cdot \vec{n}+\sum_{j=1}^{2} \alpha_{j}(t) \vec{\eta}_{j}(x) \cdot \vec{n}(x)+\alpha_{3}(t)\left(\vec{\eta}_{3}(x) \cdot \vec{n}(x)-\vec{\eta}_{3}(y) \cdot \vec{N}(y)\right)\right), \\
& y \in \mathcal{G},
\end{aligned}
$$

where $x=y+N(y) \rho(y, t)$ (we have applied the relation $\vec{\eta}_{3}(y) \cdot \vec{N}(y)=0$ ). The estimate

$$
\left\|\rho_{t}(\cdot, t)\right\|_{L_{2}(\mathcal{G})} \leq c\left(\left\|\vec{v}^{\perp}(\cdot, t)\right\|_{L_{2}\left(\Gamma_{t}\right)}+\|\rho(\cdot, t)\|_{W_{2}^{1}(\mathcal{G})}\right)
$$

follows from (4.23) and (4.21).

Recalling Korn's inequality

$$
\left\|\vec{v}^{\perp}\right\|_{W_{2}^{1}\left(\Omega_{t}\right)} \leq c\left\|S\left(\vec{v}^{\perp}\right)\right\|_{L_{2}\left(\Omega_{t}\right)},
$$

we easily verify that the first four terms in (4.17) are dominated by

$$
\left(\frac{\nu}{2}-c \gamma\right)\left\|S\left(\vec{v}^{\perp}\right)\right\|_{L_{2}\left(\Omega_{t}\right)}^{2}-c \gamma\left\|S\left(\vec{v}^{\perp}\right)\right\|_{L_{2}\left(\Omega_{t}\right)}\|\rho\|_{W_{2}^{1}(\mathcal{G})},
$$

whence

$$
E_{1}(t) \geq\left(\frac{\nu}{2}-c \gamma\right)\left\|S\left(\vec{v}^{\perp}\right)\right\|_{L_{2}\left(\Omega_{t}\right)}^{2}-c \gamma\left\|S\left(\vec{v}^{\perp}\right)\right\|_{L_{2}\left(\Omega_{t}\right)}\|\rho\|_{W_{2}^{1}(\mathcal{G})}+\gamma \delta_{0}^{2} R[\rho]-c \delta \gamma\|\rho\|_{W_{2}^{1}(\mathcal{G})}^{2} .
$$

Choosing $\delta$ and $\gamma$ sufficiently small (but independent of $t,\|\rho\|_{W_{2}^{1}(\mathcal{G})}$, and $\left\|S\left(\vec{v}^{\perp}\right)\right\|_{L_{2}\left(\Omega_{t}\right)}$ ) and using (2.6), we obtain inequality (4.18) with some $b>0$. This completes the proof of the theorem.

The following theorem concerns uniform estimates for the Hölder norms of the solution of problem (2.17).

Theorem 4.2. Under the assumptions of Theorem 1.1, the solution of problem (2.17) satisfies the estimate

$$
\begin{aligned}
\left|\vec{v}_{r t}(\cdot, t)\right|_{C^{\alpha}\left(\Omega_{t}\right)}+\left|\vec{v}_{r}(\cdot, t)\right|_{C^{2+\alpha}\left(\Omega_{t}\right)}+\left|p_{r}(\cdot, t)\right|_{C^{1+\alpha}\left(\Omega_{t}\right)} & \\
& +|\rho(\cdot, t)|_{C^{3+\alpha}(\mathcal{G})}+\left|\rho_{t}(\cdot, t)\right|_{C^{2+\alpha}(\mathcal{G})}+\left|\rho_{t t}(\cdot, t)\right|_{C^{\alpha}(\mathcal{G})} \\
\leq & c\left(\sup _{t-2 \tau_{0} \leq t^{\prime} \leq t}\left\|\vec{v}_{r}(\cdot, t)\right\|_{L_{2}\left(\Omega_{t^{\prime}}\right.}+\sup _{t-2 \tau_{0} \leq t^{\prime} \leq t}\left\|\rho\left(\cdot, t^{\prime}\right)\right\|_{W_{2}^{1}(\mathcal{G})}\right),
\end{aligned}
$$

where $\tau_{0}$ is a small number, $t>2 \tau_{0}$, and $c$ is a constant independent of $t$.

The proof of this theorem does not differ from that of Theorem 4.1 in [6], where the solution of (2.22) with $\omega=\omega_{0}$ was estimated. To obtain (4.24), we can use both the fixed and the rotating coordinate systems, due to relations (2.23) and

$$
\omega^{\prime}(t)=-\frac{2 \beta}{I^{2}(t)} \int_{\Omega_{t}}\left(v_{1} x_{1}+v_{2} x_{2}\right) d x=-\frac{2 \beta}{I^{2}(t)} \int_{\Omega_{t}}\left(v_{r 1} x_{1}+v_{r 2} x_{2}\right) d x .
$$

Therefore, inequality (4.4) in [6] is true both for $\vec{v}_{r}$ and for $\vec{w}$.

Having estimates (4.12) and (4.13) at hand, we can extend the solution to the entire infinite time interval $t \geq 0$ in precisely the same way as this was done in [6], thus completing the proof of Theorem 2.1. 


\section{REFERENCES}

[1] A. M. Lyapunov, On stability of ellipsoidal shapes of equilibrium of revolving liquid, Collected Works. Vol. 3, Akad. Nauk SSSR, Moscow, 1959, pp. 5-113. (Russian)

[2] P. Appell, Traité de mécanique rationnelle. T. 4, Fasc. I. Figures d'équilibre d'une masse liquide homogène en rotation, Gauthier-Villars, Paris, 1932.

[3] R. A. Brown and L. E. Scriven, The shape and stability of rotating liquid drops, Proc. Roy. Soc. London Ser. A 371 (1980), 331-357. MR0576833 (82m:76027)

[4] M. Padula and V. A. Solonnikov, Existence of non-steady flows of an incompressible, viscous drop of fluid in a frame rotating with finite angular velocity, Elliptic and Parabolic Problems (Rolduc/Gaeta, 2001), World Sci. Publishing, River Edge, NJ, 2002, pp. 180-203. MR1937540 (2003h:35210)

[5] V. A. Solonnikov, A generalized energy estimate in a problem with a free boundary for a viscous incompressible fluid, Zap. Nauchn. Sem. S.-Peterburg. Otdel. Mat. Inst. Steklov. (POMI) 282 (2001), 216-243. (Russian) MR.1874890(2003b:35217)

[6] - The problem of evolution of an isolated liquid mass, Sovrem. Mat. Fund. Naprav. 3 (2003), 43-62. (Russian)

[7] A. D. Myshkis (ed.), Hydromechanics of weightlessness, "Nauka", Moscow, 1976. (Russian)

[8] V. A. Solonnikov, On the justification of quasistationary approximation in the problem of motion of a viscous capillary drop, Interfaces Free Bound. 1 (1999), 125-173. MF.1867129 (2002h:35352)

[9] _ Lectures on evolution free boundary problems: classical solutions, Lecture Notes in Math., vol. 1812, Springer, Berlin, 2003, pp. 123-175. MR2011035

St. Petersburg Branch, Steklov Mathematical Institute, Russian Academy of Sciences, Fontanka 27, St. Petersburg 191023, Russia

E-mail address: solonnik@pdmi.ras.ru

Received 18/AUG/2003

Translated by I. V. DENISOVA 